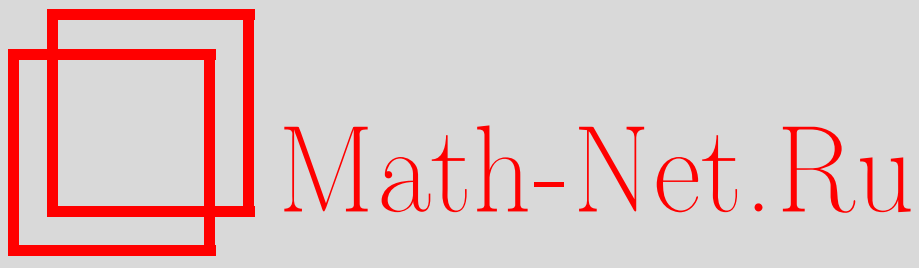

М. В. Балашов, Е. С. Половинкин, $M$-сильно выпуклые подмножества и их порождающие множества, Матем. сб., 2000, том 191, номер 1, 27-64

DOI: https://doi.org/10.4213/sm447

Использование Общероссийского математического портала Math-Net.Ru подразумевает, что вы прочитали и согласны с пользовательским соглашением

http://www . mathnet.ru/rus/agreement

Параметры загрузки:

IP: 35.173 .219 .149

26 апреля 2023 г., 13:18:05 
УДК 517.977

\author{
М.В. Балашов, Е.С. Половинкин
}

\title{
$M$-сильно вьпуклые подмножества и их порождающие множества
}

\begin{abstract}
В работе для множеств из банахова пространства вводится понятие порождающего множества $M$ и понятие $M$-сильно вьпуклого множества, представимого в виде пересечения множеств вида $M+x$, являющихся сдвигами порождающего множества $M$. Порождающее множество должно удовлетворять некоторому условию, которое обеспечивает, как показано в работе, специальный опорный принцип. На основании опорного принципа построен новьй раздел выпуклого анализа, позволяющий усиливать классические результаты типа теоремы Каратеодори или теоремы Крейна-Мильмана. Описаны различные классы порождающих множеств, изучены свойства $M$-сильно вьпуклых множеств.

Библиография: 27 названий.
\end{abstract}

\section{Введение и основные обозначения}

Понятие выпуклости играет важную роль в различных областях фундаментальной и прикладной математики. Развитие математики и расширение ее приложений привели к созданию различных аналогов и обобшений понятия выпуклости. Аксиоматический подход к понятию вьпуклости заключается в следующем. В произвольном множестве $X$ выбирается некоторое семейство подмножеств $\Phi$, называемое базой выпуклости. Множество $A$ называется $\Phi$-выпукльлм, если оно представимо в виде пересечения некоторых множеств из данного семейства $\Phi$ (см., например, [1]-[4]). Аналогично вводятся $F$-выпуклые функции, которые через надграфик можно свести к $\Phi$-выпуклым множествам (см., например, [5], [6]). Большое количество работ было посвящено исследованиям указанных классов множеств и функций (ссылки можно найти, например, в [4]).

Используя приведенное обобщение понятия выпуклости, можно не только ослаблять классическое понятие вьпуклости, но и усиливать его, получая при этом новые результаты выпуклого анализа. В работах [7]-[13] исследованы специальные классы $\Phi$-выпуклых множеств, у которых каждое семейство $\Phi$ состоит из замкнутых шаров в $\mathbb{R}^{n}$ произвольного, но фиксированного для данного семейства радиуса $R>0$ с произвольньми центрами. То есть каждое семейство $\Phi$ порождено сдвигами одного множества - шара заданного радиуса $R>0$ с центром в нуле. Как оказалось, этот специальный класс $\Phi$-выпуклых множеств, названный сильно выпуклыми множествами радиуса $R$, удовлетворяет (см. [8]-[11]) особому опорному принципу и обладает цельм рядом новых алгебраических и топологических

Работа выполнена при финансовой поддержке Российского фонда фундаментальных исследований (грант № 98-01-00645) и Конкурсного центра фундаментального естествознания (грант № 97-0-1.9-26).

(C) М.В. БАЛАшов, Е. С. ПоЛОВИнкин 
свойств для класса сильно вьпуклых множеств. Установлено специальное свойство этих классов множеств, состоящее в том, что для всякого сильно выпуклого множества радиуса $R$ найдется другое сильно выпуклое множество такое, что сумма Минковского этих двух множеств в точности равняется шару того же радиуса $R$ с центром в нуле. Естественно было бы шар с центром в нуле радиуса $R>0$ назвать порождающим множеством для данного класса $\Phi$-выпуклых множеств.

Настоящая статья является развитием работы [9] и посвящена исследованию таких классов $\Phi$-выпуклых множеств, у которых каждое семейство $\Phi$ образовано множествами, получаемыми в результате всевозможных сдвигов некоторого одного фиксированного выпуклого множества, которое обязано удовлетворять специальному свойству полного выметания.

В 1995 году в С.-Петербурге на Международной конференции "Set-valued calculus and nonsmooth analysis" после доклада Е. С. Половинкина о сильно выпуклых множествах профессор R.T. Rockafellar указал автору на то, что ему известен другой пример порождаюшего множества - надграфик параболы. Этот факт послужил дополнительным стимулом для проведения дальнейших поисков новых порождающих множеств и исследований свойств сильно выпуклых множеств.

В статье предпринята попытка описать новые классы порождаюших множеств, исследовать их общие свойства и свойства соответствующих им сильно выпуклых множеств; сформулирован опорньй принцип, характеризующий как порождающие множества, так и сильно выпуклые множества. В работе показано, что не только шар из конечномерного евклидова пространства, но и всякий шар из гильбертова пространства также является порождающим. Установлено, что прямая сумма любых порождающих множеств является порождающим множеством. Получены условия, при которых сохраняется свойство быть порождающим множеством для результирующего множества при взятии пределов от последовательности порождающих множеств или после линейных операций с порождающими множествами. Показано, что на плоскости любое выпуклое компактное множество является порождающим множеством, однако в пространствах большей размерности это уже неверно.

Введено и исследовано понятие $M$-сильно вьпуклой оболочки множества. Показано, что когда порождающее множество $M \subset \mathbb{R}^{n}$ компактно и строго выпукло, справедлив аналог теоремы Каратеодори о том, что всякая точка из $M$-сильно выпуклой оболочки компактного множества $A$ содержится в $M$-сильно выпуклой оболочке некоторого подмножества этого множества $A$, состоящего не более чем из $n+1$ точек.

Нами доказано, что надграфик квадрата нормы в гильбертовом пространстве является порождающим множеством. При этом были получены новые свойства

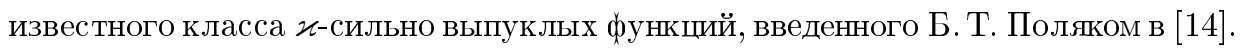

В случае, когда $M$ является шаром $B_{R}(0)$ из гильбертова пространства, для произвольного компактного множества введены понятия множеств $M$-сильно крайних точек и $M$-сильно выступающих точек, являющихся соответственно подмножествами крайних точек и выступающих точек этого множества. Нами получен аналог теоремы Крейна-Мильмана, в котором крайние точки заменены на $M$-сильно крайние точки, а выпуклая оболочка - на $M$-сильно выпуклую оболочкy. 
$\mathrm{B} \mathbb{R}^{n}$ на основе центра Штейнера и $B_{R}(0)$-сильно выпуклой оболочки множества построен новый класс непрерывных по Липшицу однозначных селекторов выпуклозначных и компактнозначных многозначных отображений.

В конце работы приведены примеры и контрпримеры, показывающие, что свойство быть порождаюшим множеством является достаточно капризным, в простых случаях (например для выпуклых многогранников) может не выполняться и легко нарушаться при алгебраических операциях с порождающими множествами.

В работе через $E$ будем обозначать рефлексивное банахово (полное линейное нормированное) пространство над вешественньм полем скаляров, через $\|\cdot\|$ - норму в $E$. Через $\mathscr{H}$ будем обозначать гильбертово (полное бесконечномерное) пространство со скалярным произведением $\langle a, b\rangle$ векторов $a$ и $b$ и согласованной с ним нормой $\|\cdot\|$. Через $\mathbb{R}^{n}$ будем обозначать $n$-мерное евклидово пространство.

Напомним, что суммой Минковского множеств $A$ и $B$ из пространства $E$ называется множество вида $A+B=\{a+b: a \in A, b \in B\}$; разностью Минковского-Понтрягина множеств $A$ и $B$ из $E$ называется множество $A * B=$ $\{x: x+B \subset A\} ;$ произведением скаляра $\lambda \in \mathbb{R}$ на множество А называется множество $\lambda A=\{\lambda a: a \in A\}$. В пространстве $E$ через $B_{R}(a)$ будем обозначать замкнутый шар $\{x:\|x-a\| \leqslant R\}$ радиуса $R>0$ с центром в точке $a$. Замыкание множества $A$ будем обозначать через $\bar{A}$, внутренность (относительную внутренность) - через $\operatorname{int} A(\operatorname{ri} A)$, границу - через $\partial A$. Через $E^{*}$ обозначим сопряженное пространство к пространству $E, B_{R}^{*}(p)=\left\{q \in E^{*}:\|q-p\|_{*} \leqslant R\right\}$. Диаметром ограниченного множества $A$ называется величина $\operatorname{diam} A=\sup \{\|x-y\|: x, y \in A\}$. Для $p \in E^{*}$ через $\langle p, x\rangle$ будем обозначать значение функционала $p$ в точке $x \in E$. Через $s(p, A), p \in E^{*}$, будем обозначать опорную функиию множества $A$, т.е. $s(p, A)=\sup \{\langle p, x\rangle: x \in A\}$. Метрика Хаусдорфа в пространстве непустых замкнутых выпуклых множеств из банахова пространства $E$ определяется по формуле $h(A, B)=\inf \left\{r>0: A \subset B+B_{r}(0), B \subset A+B_{r}(0)\right\}$.

Для выпуклого множества $A \subset E$ барьерный конус $b(A) \subset E^{*}$ и рецессивный конус $b(A)^{-} \subset E$ определяются по формулам $b(A)=\left\{p \in E^{*}: s(p, A)<+\infty\right\}$ и $b(A)^{-}=\{x \in E:\langle p, x\rangle \leqslant 0 \forall p \in b(A)\}$.

Для выпуклого замкнутого множества $A$ и для вектора $p \in b(A), p \neq 0$, через $A(p)$ будем обозначать опорное множество $A(p)=\{x \in A:\langle p, x\rangle=s(p, A)\}$. Если $A(p)$ непусто, то через $x_{p}^{A}$ будем обозначать элемент множества $A(p)$.

Пусть банахово пространство $E$ представлено в виде прямой суммы Минковского своих подпространств $E_{1}$ и $E_{2}$ (т.е. $E=E_{1}+E_{2}$ и $E_{1} \cap E_{2}=0$ ). Тогда множество $M=M_{1}+M_{2}$, где $M_{1} \subset E_{1}$ и $M_{2} \subset E_{2}$, будем называть прямой суммой множеств $M_{1}$ и $M_{2}$ и обозначать $M_{1} \oplus M_{2}$.

\section{§1. Опорный принцип}

Прежде всего дадим определения основных понятий: порождающего множества и сильно вьпуклого множества.

ОПРЕДЕЛЕНИЕ 1.1. Выпуклое замкнутое множество $M$ в банаховом пространстве $E$ назовем порожсдающим множсеством, если для любого множества $A \neq \varnothing$ вида

$$
A=\bigcap_{x \in X}(M+x)
$$


найдется выпуклое замкнутое множество $B \subset E$ такое, что

$$
\overline{A+B}=M .
$$

ОПРЕДЕЛЕНИЕ 1.2. Для всякого порождаюшего множества $M$ непустое множество вида (1.1) будем называть $M$-сильно выпуклымм множеством.

ЗАмЕчАниЕ 1.1. При определенных ограничениях на $M$ замыкание в формуле (1.2) можно убрать. Именно такого типа условия фигурируют во многих полученных далее результатах. При этом для простоты изложения мы не всегда приводим соответствуюшие условия в максимальной общности. Главным образом мы пользуемся леммой 1.1 , хотя в конкретных случаях возможны ослабления условий этой леммы.

Лемма 1.1. Пусть $A$ и $B$ - замкнутые выпуклье множества из рефлексивного банахова пространства $E$, удовлетворяющие условию $0 \in \operatorname{int}(b(A)-b(B))$. Тогда множество $A+B$ также замкнуто.

ДокаЗательство. Пусть последовательность $\left\{c_{k}\right\} \subset A+B$ такова, что $\lim _{k \rightarrow \infty} c_{k}=c$ в $E$. Тогда существуют $\left\{a_{k}\right\} \subset A$ и $\left\{b_{k}\right\} \subset B$ такие, что $c_{k}=a_{k}+b_{k}$ для всех $k$. В силу условия леммы для любого $p \in E^{*}, p \neq 0$, сушествуют $\lambda>0, p_{1} \in b(A)$ и $p_{2} \in b(B)$ такие, что $p=\lambda\left(p_{1}-p_{2}\right)$. Поэтому

$$
\begin{aligned}
\left\langle p, a_{k}\right\rangle & =\lambda\left\langle p_{1}-p_{2}, a_{k}\right\rangle=\lambda\left\langle p_{1}, a_{k}\right\rangle+\lambda\left\langle p_{2}, b_{k}\right\rangle-\lambda\left\langle p_{2}, c_{k}\right\rangle \\
& \leqslant \lambda\left(s\left(p_{1}, A\right)+s\left(p_{2}, B\right)\right)+\lambda(\|c\|+1)\left\|p_{2}\right\|_{*},
\end{aligned}
$$

т.е. $\sup _{k}\left\langle p, a_{k}\right\rangle<+\infty$ для любого $p \in E^{*}$.

По теореме Банаха-Штейнгауза последовательность $\left\{a_{k}\right\}$ ограничена. Поэтому она относительно слабо компактна в $E$, т.е. сушествует подпоследовательность $\left\{a_{k m}\right\}$, которая слабо сходится к $a$, при этом подпоследовательность $b_{k m} \subset B$ слабо сходится к $c-a$. Так как $A$ и $B$ выпуклы и замкнуты, то они слабо замкнуты, т.е. $a \in A, c-a \in B$, значит, $c \in A+B$.

Отметим некоторые свойства барьерных конусов.

ПРЕДЛОЖЕНИЕ 1.1 (см. [15; гл. 1, 5]).

1) Ecлu $A, B \subset E, A \subset B, \operatorname{mo} b(A) \supset b(B), b(A)=b(\bar{A})$;

2) если $A, B \subset E$, то $b(A+B)=b(A) \cap b(B)$;

3) если $A, B \subset E$ замкнуты и выпукль, причем $0 \in \operatorname{int}(A-B)$, то $b(A \cap B)=b(A)+b(B)$.

Лемма 1.2. Пусть $A$ - замкнутое выпуклое множество из рефлексивного банахова пространства $E$. Тогда для любого $p \in \operatorname{int} b(A)$ опорное множество $A(p)=\{x \in A:\langle p, x\rangle=s(p, A)\}$ есть непустое замкнутое выпуклое подмножество множества $A$. 
ДокаЗАтЕльство. Фиксируем $p \in \operatorname{int} b(A)$. Определим множество $A_{1}=$ $\{x \in A:\langle p, x\rangle \geqslant s(p, A)-1\}$. В силу определения опорной функции множество $A_{1}$ непусто, выпукло и замкнуто, причем $s\left(p, A_{1}\right)=s(p, A)$. Покажем, что $s\left(p, A_{1}\right)<+\infty$ для всех $p \in E^{*}$, т.е. $b\left(A_{1}\right)=E^{*}$. В самом деле, $A_{1}=A \cap H$, где $H=\{x \in E:\langle-p, x\rangle \leqslant 1-s(p, A)\}$. Следовательно, $-p \in b(H)$, int $H \neq \varnothing$ и $0 \in \operatorname{int}(H-A)$. По свойству 3$)$ предложения $1.1 b\left(A_{1}\right)=b(A \cap H)=b(A)+b(H)$.

Так как для некоторого $\delta>0$ верно $p+B_{\delta}^{*}(0) \subset b(A)$, то $B_{\delta}^{*}(0) \subset b(A)+b(H)$, т.е. $b(A)+b(H)=E^{*}$.

Следовательно, можем считать, что минимизирующая последовательность $\left\{a_{k}\right\} \subset A_{1}$ такая, что $\lim _{k \rightarrow \infty}\left\langle p, a_{k}\right\rangle=s(p, A)$, сходится слабо к некоторой точке $a$, а так как $A_{1}$ замкнуто и выпукло, т.е. слабо замкнуто, то $a \in A(p)$.

Простьм следствием теорем об отделимости (см., например, [16; теорема 3.1.2]) является

ПрЕДЛОЖЕНИЕ 1.2. Пусть $A$ - выпуклое замкнутое подмножество из банахова пространства $E$ u int $b(A) \neq \varnothing$. Тогда

$$
A=\bigcap_{\substack{\|p\|_{*}=1 \\ p \in \operatorname{int} b(A)}} H_{p}
$$

ฉде

$$
H_{p}=\{x \in E:\langle p, x\rangle \leqslant s(p, A)\}
$$

ЗАмечАние 1.2 . В случае, когда $E=\mathbb{R}^{n}$, формулу (1.3) можно усилить, заменив в ней int $b(A)$ на $\operatorname{ri} b(A)$, при этом отказавшись в предложении 1.2 от условия, что $\operatorname{int} b(A)$ непусто.

Уточним некоторые свойства барьерного и рецессивного конусов неограниченного выпуклого множества $A \subset E$.

Как показано в [15; предложение 1.5.3], для любого замкнутого выпуклого множества $A \subset E$ и для любого $a \in A$ справедливо равенство

$$
b(A)^{-}=\bigcap_{\lambda>0} \lambda(A-a)
$$

Лемма 1.3. Пусть $A$ и $M$ - непустые выпуклые замкнутые множества из $E$, причем $A=\bigcap_{x \in X}(M+x)$ при некотором $X$. Тогда справедливы формуль

$$
\begin{aligned}
& b(M)^{-}=M * * M, \\
& b(A)^{-}=b(M)^{-}, \\
& \overline{b(A)}=\overline{b(M)}, \quad \operatorname{int} b(A)=\operatorname{int} b(M) .
\end{aligned}
$$


ДоказАТЕльство. Докажем равенство (1.6). Из формулы (1.5) следует, что $b(M)^{-} \subset M-x \forall x \in M$, т.е. $b(M)^{-} \subset M \stackrel{*}{*}^{-}$. Аналогично случаю $\mathbb{R}^{n}$ легко показать, что множество $M \stackrel{*}{*}$ есть вьпуклый конус (см. [17; теорема 2.8.1]). Допустим, что найдется точка $x_{0} \in M \stackrel{*}{-} M$ такая, что $x_{0} \notin b(M)^{-}$. Тогда по теореме об отделимости существует функционал $p_{0} \in E^{*}, p_{0} \neq 0$, такой, что $\left\langle p_{0}, x_{0}\right\rangle>s\left(p_{0}, b(M)^{-}\right)$. Это означает, что $\left\langle p_{0}, x\right\rangle \leqslant 0 \forall x \in b(M)^{-}$, т.е. $p_{0} \in \overline{b(M)}$. $\mathrm{C}$ другой стороны, это означает, что $\left\langle p_{0}, x_{0}\right\rangle>0$, и так как $x_{0}$ принадлежит конусу $M \stackrel{*}{*} M$, то $p_{0} \in \operatorname{int}\left(E^{*} \backslash b(M)\right)$. Противоречие приводит к (1.6).

Докажем (1.7). Из включения $A \subset M+x$ следует, что $b(A) \supset b(M)$, откуда, в свою очередь, следует включение $b(A)^{-} \subset b(M)^{-}$. Пусть $x \in b(M)^{-}$, тогда по (1.6) $x+M \subset M$, т.е. $x+M+y \subset M+y$ для каждого $y \in X$, следовательно $\bigcap_{y \in X}(x+M+y) \subset \bigcap_{y \in X}(M+y)$, т.е. $x+A \subset A$, откуда $x \in b(A)^{-}$в силу (1.6).

Первое равенство в (1.8) следует из (1.7) в силу определения рецессивного конуса как поляры к барьерному конусу, второе равенство вытекает из первого в силу свойств выпуклых множеств.

Лемма 1.4. Пусть $A, B$ и $M$ - такие выпуклье замкнутые множества из рефлексивного банахова пространства $E$, что int $b(M) \neq \varnothing u A+B=M$. Тогда $A(p)+B(p)=M(p)$ для любого $p \in \operatorname{int} b(M)$.

ДокАЗАТЕльство. В силу лемм 1.2, 1.3 и свойства 2) из предложения 1.1 для любого $p \in \operatorname{int} b(M)$ множества $A(p), B(p), M(p)$ непусты.

1. Пусть $a \in A(p), b \in B(p)$. Тогда $a+b \in M$ и

$$
\langle p, a+b\rangle=s(p, A)+s(p, B)=s(p, M),
$$

т.е. $a+b \in M(p)$, откуда следует $A(p)+B(p) \subset M(p)$.

2. Пусть $z \in M(p)$ и $a \in A, b \in B$ такие, что $z=a+b$. Допустим, что $a \notin A(p)$. Тогда

$$
s(p, M)=\langle p, z\rangle=\langle p, a+b\rangle\langle s(p, A)+s(p, B)=s(p, M)
$$

получили противоречие. Следовательно, справедливо включение $A(p)+B(p) \supset$ $M(p)$.

Tеорема 1.1. Пусть $M \subset E-$ порождающее множество $u$ int $b(M) \neq \varnothing$. Тогда для любого $M$-сильно выпуклого множества $A$, для любого $p \in \operatorname{int} b(M)$, $\|p\|_{*}=1$, и любого $x_{p}^{A} \in A(p)$ существуют $x_{p}^{M} \in M(p)$ такие, что

$$
A=\bigcap_{\substack{\|p\|_{*}=1 \\ p \in \operatorname{int} b(M)}}\left(M+x_{p}^{A}-x_{p}^{M}\right)
$$

Обратно, если выпуклое замкнутое множество $M$ таково, ито int $b(M) \neq \varnothing$ и любое множсество А вида (1.1) представимо по формуле (1.9), то множество $M-$ порожсдающее. 
ДокаЗАТЕльство. Для любого $A$ вида (1.1) int $b(A)=\operatorname{int} b(M)$ в силу леммы 1.3.

1. Пусть $M$ - порождающее множество и $A$ имеет вид (1.1). По определению 1.1 существует выпуклое замкнутое множество $B$ такое, что $\overline{A+B}=M$. Так как $b(B) \supset b(M)$, то по лемме 1.1 получаем, что $A+B=M$. Фиксируем произвольный вектор $p \in \operatorname{int} b(M),\|p\|_{*}=1$, и произвольньй $x_{p}^{A} \in A(p)$. Фиксируем любой $x_{p}^{B} \in B(p)$. Для любых $x_{p}^{A}$ и $x_{p}^{B}$ в силу леммы 1.4 сумма $x_{p}^{A}+x_{p}^{B} \in M(p)$, поэтому обозначим через $x_{p}^{M}$ выражение

$$
x_{p}^{A}+x_{p}^{B}=x_{p}^{M}=x_{p}^{M}\left(x_{p}^{A}\right) \in M(p) .
$$

Из равенства $A+B=M$ и включения $x_{p}^{B} \in B$ следует включение $A+x_{p}^{B} \subset M$, отсюда и из (1.10) получаем $A \subset M+x_{p}^{A}-x_{p}^{M}\left(x_{p}^{A}\right)$. Так как последнее включение верно для любых $p \in \operatorname{int} b(A),\|p\|_{*}=1$, и любых $x_{p}^{A} \in A$, то

$$
A \subset \bigcap_{\substack{\|p\|_{*}=1 \\ p \in \operatorname{int} b(M)}}\left(M+x_{p}^{A}-x_{p}^{M}\left(x_{p}^{A}\right)\right) .
$$

Пусть $H_{p}=\{x \in E:\langle p, x\rangle \leqslant s(p, A)\}$. Ясно, что $M+x_{p}^{A}-x_{p}^{M}\left(x_{p}^{A}\right) \subset H_{p}$ для всех $p \in \operatorname{int} b(M)$. В силу выпуклости и замкнутости $A$ имеем по предложению 1.2

$$
\bigcap_{\substack{\|p\|_{*}=1 \\ p \in \operatorname{int} b(M)}}\left(M+x_{p}^{A}-x_{p}^{M}\left(x_{p}^{A}\right)\right) \subset \bigcap_{\substack{\|p\|_{*}=1 \\ p \in \operatorname{int} b(M)}} H_{p}=A
$$

Равенство (1.9) доказано.

2. Пусть для любого $A$ вида (1.1) имеет место формула (1.9). Покажем, что множество $M-$ порождающее.

Фиксируем $q \in \partial B_{1}^{*}(0) \cap \operatorname{int} b(M)$. Тогда

$$
s(q, A) \leqslant \inf _{\substack{\|p\|_{*}=1 \\ p \in \operatorname{int} b(M)}} s\left(q, M+x_{p}^{A}-x_{p}^{M}\right) \leqslant s\left(q, M+x_{q}^{A}-x_{q}^{M}\right)=s(q, A),
$$

откуда следует, что все неравенства здесь надо заменить на равенства, т.е.

$$
s(q, A)=\inf _{\substack{\|p\|_{*}=1 \\ p \in \operatorname{int} b(M)}} s\left(q, M+x_{p}^{A}-x_{p}^{M}\right)=s(q, M)-\sup _{\substack{\|p\|_{*}=1 \\ p \in \operatorname{int} b(M)}}\left\langle q, x_{p}^{M}-x_{p}^{A}\right\rangle .
$$

Определим множество

$$
B=\overline{\mathrm{co}} \bigcup_{\substack{\|p\|_{*}=1 \\ p \in \operatorname{int} b(M)}}\left(x_{p}^{M}\left(x_{p}^{A}\right)-x_{p}^{A}\right) .
$$

Тогда в силу (1.11) для любого $q \in \partial B_{1}^{*}(0) \cap \operatorname{int} b(M)$ выполнено равенство $s(q, A)+s(q, B)=s(q, M)$. Отсюда $A+B=M$ в силу предложения 1.2 и леммы 1.1.

СЛЕДСТВИЕ 1.1 (опорный принцип). Пусть $M$ есть выпуклое замкнутое множество в $E$, причем int $b(M) \neq \varnothing$. Множсество $M$ является порожсдающим тогда и только тогда, когда для любого множсества $A \neq \varnothing$ вида (1.1), для любого $p \in \partial B_{1}^{*}(0) \cap \operatorname{int} b(M)$ и любого $x_{p}^{A} \in A(p)$ найдутся $x_{p}^{M} \in M(p)$ такие, что

$$
A \subset M+x_{p}^{A}-x_{p}^{M} .
$$

Доказательство непосредственно следует из равенства (1.9). 


\section{§ 2. О некоторых классах порождающих множеств}

Приступим к исследованию задачи нахождения классов порождающих множеств и к изучению операций с порождающими множествами, не выводящих из класса порождающих множеств.

Мы знаем, что класс порождающих множеств непуст. Так, например, в работе [9] впервые показано, что всякий шар $B_{R}(a)$ из $\mathbb{R}^{n}$ является порождающим множеством. Легко убедиться в том, что всякий $k$-мерньй симплекс в $\mathbb{R}^{n}$ также является порождающим множеством (см. пример 9.3).

В то же время нам известны примеры, показывающие, что даже в евклидовом пространстве $\mathbb{R}^{n}$ при $n>2$ достаточно простые множества типа пирамиды (см. пример 9.1) или веретена (см. пример 9.2) не являются порождаюшими множествами.

Лемма 2.1. Пусть $M \subset E$ - въпуклое замкнутое множество с непустой внутренностью такое, что int $b(M) \neq \varnothing$. Пусть для любого множества $X$ такого, что $\operatorname{int}(M * X) \neq \varnothing$, найдется выпуклое множество $B_{X}$, для которого $(M \stackrel{*}{*})+B_{X}=M$. Тогда множсество $M$ является порожсдаюшим.

ДоказАтельство. Пусть $X-$ такое множество, что $\operatorname{int}(M \stackrel{*}{X})=\varnothing$, но $M \stackrel{*}{ } X \neq \varnothing$. Покажем, что найдется множество $B$ такое, что $(M \stackrel{*}{*})+B=M$. Без ограничения общности можем считать, что $0 \in X \subset M$. Поскольку $M * X=$ $M * \overline{c o} X$, то без ограничения общности полагаем множество $X$ выпукльм и замкнутым.

Так как $0 \in X \subset M$ и множество $X$ выпукло, то $\forall \lambda \in(0,1) \lambda X \subset X$ и $M \stackrel{*}{\lambda} X \supset(1-\lambda) M$, т.е. $\operatorname{int}(M \stackrel{*}{ } \lambda X) \neq \varnothing \forall \lambda \in(0,1)$. Пусть $\lambda_{n}=(n-1) / n$, $A_{n}=M \stackrel{*}{ } \lambda_{n} X$. Очевидно, $A_{n} \supset A_{n+1} \supset M \stackrel{*}{ }$. П. Пусть $A=\bigcap_{n=1}^{\infty} A_{n}$. Тогда $M \stackrel{*}{X} \subset A$. В свою очередь, если $z \in A$, то $z+\lambda_{n} X \subset M$, т.е. $\forall x \in X$ $z+\lambda_{n} x \in M$ для всех $n$, следовательно, $z+x \in M$ для всех $x$, т.е. $z \in M * X$. Итак, $A=M * * X$. По условию леммы найдутся выпуклые замкнутые множества $B_{n}$ такие, что $A_{n}+B_{n}=M$. При этом $A+B_{n} \subset M ; B_{n} \subset B_{n+1}$ для всех натуральных $n$.

Рассмотрим $B=\overline{\bigcup_{n=1}^{\infty} B_{n}}$. Тогда $A+B \subset M$. Докажем обратное включение. Пусть $m \in M$, тогда найдутся $a_{n} \in A_{n}$ и $b_{n} \in B_{n}$ такие, что $m=a_{n}+b_{n}$. Так как $\overline{b\left(A_{n}\right)}=\overline{b(M)} \subset \overline{b\left(B_{n}\right)}$, то, рассуждая, как в доказательстве леммы 1.1, получаем, что $\left\{a_{n}\right\}$ и $\left\{b_{n}\right\}$ с точностью до подпоследовательности слабо сходятся к $a$ и $m-a$ соответственно; так как $\left\{b_{n}\right\} \subset B$ и множество $B$ выпукло и замкнуто, то $m-a \in B$. Поскольку для любого натурального $N$ верно $\left\{a_{n}\right\} \subset A_{N}$ при всех $n>N$, то $a \in A_{N}$, т.е. $a \in A$. В итоге, $m \in A+B$, т.е. $A+B=M$.

ТЕОРема 2.1. Пусть в сепарабельном банаховом пространстве Е дань ограниченные замкнутые выпуклые множества $M u M_{k}, k=1,2, \ldots$, причем $M_{k}$ суть порождающие множества, для которых выполнено одно из двух условий:

1) $h\left(M_{k}, M\right) \rightarrow 0$, int $M \neq \varnothing$;

2) $M_{k} \supset M \forall k u M_{k} \rightarrow M$ в слабой топологии.

Тогда $M$ также будет порождающим множеством. 
ДоказАтельство. 1. Пусть int $M \neq \varnothing$ и $h\left(M_{k}, M\right) \rightarrow 0$. По лемме 2.1 достаточно проверить, что для любого множества $X$ такого, что int $(M \stackrel{*}{*}) \neq \varnothing$, найдется $B$ такое, что $(M \stackrel{*}{X)}+B=M$.

Так как $\operatorname{int}(M \stackrel{*}{X}) \neq \varnothing$, то существует шар $B_{\alpha}(z) \subset M \stackrel{*}{X, \alpha>0 .}$ Будем рассматривать такие большие $k$, что $h=h\left(M_{k}, M\right) \leqslant \alpha$. Следовательно, $M \stackrel{*}{ } B_{h}(0) \subset M_{k}$, откуда

$$
M \stackrel{*}{X} \stackrel{*}{ } B_{h}(0) \subset M_{k} \stackrel{*}{X} .
$$

Пусть $G=M \stackrel{*}{X}$. Введем $D=\operatorname{diam} G$. Рассмотрим $x \in \partial G$. Выберем $g \in[x, z]$ так, чтобы $\|x-g\| /\|x-z\|=h / \alpha$. В силу вьпуклости $G$ получаем $(1-h / \alpha) x+$ $h / \alpha B_{\alpha}(z) \subset G$, т.е. $B_{h}(g) \subset G$. Отсюда $g \in G \stackrel{*}{*} B_{h}(0)$ и $\|x-g\| \leqslant D h / \alpha$, т.е.

$$
h\left(G, G \stackrel{*}{*} B_{h}(0)\right)=\sup _{x \in \partial G} \varrho\left(x, G * B_{h}(0)\right) \leqslant \frac{D}{\alpha} h .
$$

Взяв $l=D / \alpha$, получим

$$
M \stackrel{*}{X} \subset(M \stackrel{*}{ } X) \stackrel{*}{ } B_{h}(0)+l B_{h}(0) \subset M_{k} * \underset{*}{ } X+l B_{h}(0) .
$$

Последняя формула означает $\varepsilon$-полунепрерывность снизу $M_{k} * X$, откуда следует полунепрерывность снизу в слабой топологии $E$. Отсюда же вытекает, что при всех достаточно больших $k$ (когда $l h<\alpha) \operatorname{int}\left(M_{k} * X\right) \neq \varnothing$.

Поскольку $M_{k} \rightarrow M$ в слабой топологии $E$, то $M_{k} * \underset{X}{X}$ слабо полунепрерьвно сверху.

В итоге, $M_{k} *$ $X \rightarrow M \stackrel{*}{X}$ в слабой топологии $E$.

Для каждого $k$ найдется $B_{k}$ такое, что $\left(M_{k} * * X\right)+B_{k}=M_{k}$. Отсюда следует, что

$$
s\left(p, M_{k}\right) \rightarrow s(p, M), \quad s\left(p, M_{k} * X\right) \rightarrow s(p, M \stackrel{*}{*} X),
$$

тогда $s\left(p, B_{k}\right) \rightarrow g(p)=s(p, M)-s(p, M * X)$ - выпуклая непрерывная функция. Для $B=\left\{x:\langle p, x\rangle \leqslant g(p) \forall p \in \partial B_{1}^{*}(0)\right\}$ получаем $(M$ * $X)+B=M$.

2. Пусть $M_{k} \supset M \forall k$ и $M_{k} \rightarrow M$ в слабой топологии. Тогда $M_{k} * \underset{X}{\rightarrow} M \stackrel{*}{=}$ в слабой топологии $E$. Далее доказательство повторяет п. 1.

СлЕДСТВИЕ 2.1. Пусть в $\mathbb{R}^{n}$ дань компакт $M$ и последовательность порождающих множсеств $M_{k}$, причем $M_{k} \subset M$ для всех $k$ u $h\left(M_{k}, M\right) \rightarrow 0$. Тогда $M$ также будет порожддающим множеством.

ДокАЗАТЕльство. Переходя в несущее подпространство $M$, можем без ограничения общности считать, что int $M \neq \varnothing$ и все $M_{k}$ целиком содержатся в этом несущем подпространстве. Утверждение следует из теоремы 2.1 .

ЗАМЕчАНИЕ 2.1. В конце работы приведен пример 9.4, показывающий, что условия теоремы 2.1 сушественны.

Tеорема 2.2. Пусть $M-$ порождающее множество из Е. Тогда для любого $p \in \operatorname{int} b(M)$ опорное множсество $M(p)$ будет порождающим в многообразии $H_{p}^{\circ}=\{x:\langle p, x\rangle=s(p, M)\}$. 
ДокаЗАТЕЛЬСТво. Фиксируем $p \in \operatorname{int} b(M)$. Сдвигая при необходимости $M$, можем считать, что $H_{p}^{\circ}$ - подпространство пространства $E$. По лемме 1.2 множество $M(p)$ есть непустое замкнутое выпуклое множество. Пусть $X$ - замкнутое множество из $H_{p}^{\circ}$ и пусть

$$
A=\bigcap_{x \in X}(M(p)+x) \neq \varnothing .
$$

Очевидно, что $A(p)=A$. Определим $A_{1}=\bigcap_{x \in X}(M+x)$. Из справедливости для каждого $x \in X$ включения $A_{1}(p) \subset(M+x)(p)=M(p)+x$ следует включение

$$
A_{1}(p) \subset \bigcap_{x \in X}(M(p)+x)=A \subset A_{1}
$$

т.е. $A_{1}(p)=A$ и $A_{1} \neq \varnothing$.

Так как $M$ порождающее, то найдется выпуклое замкнутое множество $C_{1}$ такое, что $A_{1}+C_{1}=M$. По лемме 1.4 получаем, что

$$
A_{1}(p)+C_{1}(p)=M(p),
$$

т.е. $A+B=M(p)$, где в качестве $B$ возьмем $C_{1}(p)$.

Теорема 2.3. Пусть $M \subset E_{1}$ - порождающее множество и $T$ - непрерывный линейный оператор из $E_{1}$ на $E_{2}$, имеющий обратный. Тогда $T M$ является порождающим множеством в $E_{2}$.

ДоказАтЕЛЬСтво. Пусть $A_{2}=\bigcap_{x \in X}(T M+x)$. Отметим, что для любых замкнутых множеств $P \subset E_{1}$ и $Q \subset E_{2}$ образы $T P$ и $T^{-1} Q$ являются замкнутьми множествами в $E_{2}$ и $E_{1}$ соответственно в силу непрерывности операторов $T$ и $T^{-1}$ (см. [18; следствие 2.2]). Поэтому

$$
A_{2}=\bigcap_{x \in X}(T M+x)=\bigcap_{x \in X} T\left(M+T^{-1} x\right)=\bigcap_{y \in Y} T(M+y),
$$

где через $Y$ обозначили $T^{-1} X$. Обозначим $A_{1}=\bigcap_{y \in Y}(M+y)$. Покажем, что $A_{2}=T A_{1}$. Так как включение $A_{2} \supset T A_{1}$ очевидно, то докажем включение $A_{2} \subset T A_{1}$. Пусть $z \in A_{2}$. Тогда $z \in T(M+y) \forall y \in Y$, что равносильно включению $T^{-1} z \in M+y \forall y \in Y$. Поэтому $T^{-1} z \in A_{1}$, т.е. $z \in T A_{1}$. Равенство доказано.

Так как $A_{1}$ есть $M$-сильно выпуклое множество, то найдется выпуклое множество $B_{1}$ такое, что $A_{1}+B_{1}=M$. Действуя линейным оператором $T$ на обе части последнего равенства, получаем $A_{2}+T B_{1}=T M$, где $T B_{1}$ - выпуклое замкнутое множество; т.е. $T M$ - порождаюшее множество.

СлЕДСТВИЕ 2.2. Любой әллипсоид в $\mathbb{R}^{n}$ есть порождающее множество.

ДокАЗАТЕЛЬСТво. Любой эллипсоид есть образ шара при невырожденном линейном преобразовании. У тверждение следует из теоремы 2.3 и того, что любой шар из $\mathbb{R}^{n}$ - порождаюшее множество (см. [9; теоремы 1,2$\left.]\right)$. 
ЗАмЕчАниЕ 2.2. В конце работы приведен пример 9.5, показываюший, что если в условии теоремы 2.3 оператор $T$ вырожден, то утверждение этой теоремы может не выполняться.

Tеорема 2.4. Пусть $M_{1} \subset E_{1}$ u $M_{2} \subset E_{2}$ - порождающие множества. Тогда их прямая сумма $M_{1} \oplus M_{2}$ также будет порохсдаюшим множеством в прямой сумме пространств $E_{1} \oplus E_{2}$.

ДоказАтельство. Пусть $M=M_{1} \oplus M_{2}, X \subset E_{1} \times E_{2}: M * X \neq \varnothing$. Пусть $\pi_{1}-$ проектор на $E_{1}$, а $\pi_{2}-$ проектор на $E_{2}$. Докажем равенство

$$
M \stackrel{*}{ } X=M \stackrel{*}{*}\left(\pi_{1} X \oplus \pi_{2} X\right) .
$$

Правое множество в формуле (2.1) очевидно содержится в левом, поскольку $X \subset$ $\pi_{1} X \oplus \pi_{2} X$. Покажем справедливость обратного включения. Пусть $z \in M \stackrel{*}{X}$. Это равносильно включению $z+X \subset M$, действуя на которое проекторами $\pi_{1}$ и $\pi_{2}$, получаем

$$
\pi_{i} z+\pi_{i} X \subset M_{i}, \quad i=1,2 .
$$

Взяв прямую сумму двух последних включений, имеем

$$
\left(\pi_{1} z \oplus \pi_{2} z\right)+\left(\pi_{1} X \oplus \pi_{2} X\right) \subset M
$$

а так как $z=\pi_{1} z \oplus \pi_{2} z$, то $z \in M \stackrel{*}{*}\left(\pi_{1} X \oplus \pi_{2} X\right)$. Аналогично равенству (2.1) легко показать, что

$$
M \stackrel{*}{ }\left(\pi_{1} X \oplus \pi_{2} X\right)=\left(M_{1} \stackrel{*}{ } \pi_{1} X\right) \oplus\left(M_{2} \stackrel{*}{ } \pi_{2} X\right) .
$$

Пусть $B_{i}$ - такие множества, что $\left(M_{i} \stackrel{*}{*} \pi_{i} X\right)+B_{i}=M_{i}, i=1,2$. Взяв прямую сумму двух последних равенств и учитьвая равенство (2.1) и формулу (2.2), получаем, что

$$
M=(M \stackrel{*}{ } X)+\left(B_{1} \oplus B_{2}\right) .
$$

ЗАмечАниЕ 2.3. В примере 9.6 показано, что в общем случае сумма Минковского двух порождающих множеств может не быть порождающим множеством.

СлЕДСТВИЕ 2.3. Теорема 2.4 справедлива и для множеств, представимых в виде прямой суммы произвольного числа (конечного или бесконечного) порождающих множеств в соответствующей прямой сумме (конечной или бесконечной) пространств.

ДоКАЗАТЕльство. Для обобщения теоремы 2.4 в случае бесконечного числа прямых слагаемых достаточно повторить рассуждения и воспользоваться очевидньгм равенством

$$
\bigoplus_{i=1}^{\infty}\left(A_{i}+B_{i}\right)=\bigoplus_{i=1}^{\infty} A_{i}+\bigoplus_{i=1}^{\infty} B_{i}
$$

где $A_{i} \subset E_{i}$ и $B_{i} \subset E_{i}$ для всех $i$ и $\left\{E_{i}\right\}_{i=1}^{\infty}$ есть набор соответствующих векторных пространств. 
СЛЕДСТВИЕ 2.4. Гилъбертов кирпич из пространства $l_{p}$, әде $p \in(1,+\infty)$, является порождающим множеством.

ДокаЗАтЕльство. Для всякого $x \in l_{p}$ через $\pi_{i} x=x_{i}$ обозначается $i$-й элемент последовательности $x$. Гильбертовым кирпичом, как известно, называется множество $M=\left\{x \in l_{p}:\left|x_{i}\right| \leqslant 1 / i\right\}$. Отметим, что $l_{p} \subset \bigotimes_{i=1}^{\infty} \mathbb{R}_{i}$, где $\mathbb{R}_{i}=\mathbb{R}$ для Bcex $i$.

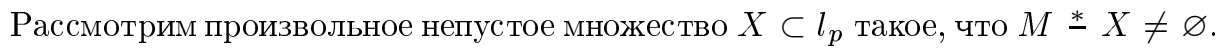
Без ограничения общности считаем, что $X \subset M$.

Поскольку для любого $i$ множества $\pi_{i} M$ суть отрезки в $\mathbb{R}_{i}$ и $\pi_{i} X \subset$ $\pi_{i} M \subset[-1 / i, 1 / i]$, то очевидно, что найдутся отрезки $B_{i} \subset \mathbb{R}_{i}$, для которых $\left(\pi_{i} M \stackrel{*}{*} \pi_{i} X\right)+B_{i}=\pi_{i} M$ и $B_{i} \subset[-3 / i, 3 / i]$. Введем множество

$$
B=\bigoplus_{i=1}^{\infty} B_{i}
$$

По следствию 2.3 справедливо равенство

$$
M \stackrel{*}{*} X+B=M
$$

Осталось показать, что $B \subset l_{p}$. В самом деле, если $b=\left(\ldots, b_{i}, \ldots\right) \in B \subset$ $\bigotimes_{i=1}^{\infty} \mathbb{R}_{i}$, то $-3 / i \leqslant b_{i} \leqslant 3 / i$, откуда следует, что

$$
\|b\|_{p}^{p}=\sum_{i=1}^{\infty}\left|b_{i}\right|^{p} \leqslant \sum_{i=1}^{\infty} \frac{3^{p}}{i^{p}}<+\infty
$$

СЛЕДСТВИЕ 2.5. Единичный шар в $l_{\infty}$ является порождающим множеством.

ДокАЗАТЕЛЬСтво. Напомним, что единичньм шаром в пространстве $l_{\infty} \subset$ $\bigotimes_{i=1}^{\infty} \mathbb{R}_{i}$ является множество $M=\left\{x \in l_{\infty}:\left|x_{i}\right| \leqslant 1\right\}$.

Повторяя рассуждения из доказательства следствия 2.4, получаем, что если $X \subset M$, то существует множество вида (2.4), для которого в силу теоремы 2.4 справедливо равенство (2.5). При этом $B_{i} \subset[-3,3]$, откуда $\|b\|_{\infty} \leqslant 3$ для любого $b \in B$, т.е. $B \subset l_{\infty}$.

TЕОРема 2.5. Пусть $M$ - выпуклый компакт из $\mathbb{R}^{n}$. Пусть для каждого $p \in \partial B_{1}(0)$, для любих наборов векторов $\left\{p_{i}\right\}_{i=1}^{k}$ и чисел $\left\{\lambda_{i}\right\}_{i=1}^{k}$ таких, что $\left\|p_{i}\right\|=1, \lambda_{i}>0, \sum_{i=1}^{k} \lambda_{i} p_{i}=p, 2 \leqslant k \leqslant n, u$ для любых $x_{p_{i}}^{M} \in M\left(p_{i}\right)$ найдутся $x_{p}^{M} \in M(p)$ mакие, что

$$
\bigcap_{i=1}^{k}\left(M-x_{p_{i}}^{M}\right) \subset M-x_{p}^{M}
$$

Тогда множество $M$ является порохсдающим. 
ДокАЗАТЕЛЬСтво. Без ограничения общности будем считать, что int $M \neq \varnothing$. Мы покажем, что для любого множества вида $A=\bigcap_{x \in X}(M+x)$, где $X$ такой компакт, что int $A \neq \varnothing$, выполнена формула (1.9). Тогда по теореме 1.1 и лемме 2.1 множество $M$ будет порождающим.

Известно, что опорная функция множества $A$ имеет вид $s(p, A)=\operatorname{co} f(p)$, где $f(p)=\min _{x \in X}(s(p, M)+\langle p, x\rangle)$. Из условия непустоты внутренности $A$ следует (см. [19; гл. $1, \S 5$, предложение 14$]$ ), что инфинум в операции овыпукления $f$ достигается, т.е.

$$
\operatorname{co} f(p)=\sum_{i=1}^{k} \lambda_{i} f\left(p_{i}\right), \quad \lambda_{i}>0, \quad\left\|p_{i}\right\|=1, \quad \sum \lambda_{i} p_{i}=p, \quad k \leqslant n .
$$

Фиксируем $x_{i} \in \operatorname{Arg} \min _{x \in X}\left\langle p_{i}, x\right\rangle, 1 \leqslant i \leqslant k$. Для любого $p \in \partial B_{1}(0)$ и любого $x_{p}^{A} \in A \subset M+x_{i}$ (при всех $i$ ) получаем $\left\langle p_{i}, x_{p}^{A}\right\rangle \leqslant s\left(p_{i}, M\right)+\left\langle p_{i}, x_{i}\right\rangle$. Тогда

$$
s(p, A)=\sum_{i=1}^{k} \lambda_{i}\left(s\left(p_{i}, M\right)+\left\langle p_{i}, x_{i}\right\rangle\right) \geqslant \sum_{i=1}^{k} \lambda_{i}\left\langle p_{i}, x_{p}^{A}\right\rangle=\left\langle p, x_{p}^{A}\right\rangle=s(p, A) .
$$

Таким образом, последние неравенства нужно заменить на равенства и $\left\langle p_{i}, x_{p}^{A}\right\rangle=$ $s\left(p_{i}, M\right)+\left\langle p_{i}, x_{i}\right\rangle$ для всех $i$, а в силу включения $x_{p}^{A} \in M+x_{i}$ найдутся $x_{p_{i}}^{M} \in M$, зависящие от $x_{p}^{A}$, такие, что $x_{p}^{A}=x_{p_{i}}^{M}+x_{i}$.

Имеем $A \subset \bigcap_{i=1}^{k}\left(M+x_{p}^{A}-x_{p_{i}}^{M}\right)$. По условию теоремы существует элемент $x_{p}^{M} \in M$ такой, что правое множество содержится в $M+x_{p}^{A}-x_{p}^{M}$. Отсюда

$$
A \subset \bigcap_{\|p\|=1}\left(M+x_{p}^{A}-x_{p}^{M}\right)
$$

Лемма 2.2. Пусть $M \subset \mathbb{R}^{2}$ есть выпукльй компакт. Пусть для всякого вектора $p \in \partial B_{1}(0)$ выбраны произвольные конечные наборы векторов $\left\{p_{i}\right\}_{i=1}^{k}$ и положительных чисел $\left\{\lambda_{i}\right\}_{i=1}^{k}$ такие, что $k \geqslant 2,\left\|p_{i}\right\|=1, p_{i} \neq p$ для всех $i$ $u \sum_{i=1}^{k} \lambda_{i} p_{i}=p$. Пусть также фиксировань произвольные $x_{p}^{M} \in M(p) u$ $x_{p_{i}}^{M} \in M\left(p_{i}\right)$. Тогда справедливо включение

$$
\bigcap_{i=1}^{k}\left(M-x_{p_{i}}^{M}\right) \subset M-x_{p}^{M}
$$

ДокАЗАТЕЛьство. Если для некоторого номера $i$ верно равенство $x_{p_{i}}^{M}=x_{p}^{M}$, то включение очевидно. Поэтому далее будем считать, что $x_{p_{i}}^{M} \neq x_{p}^{M}$ для всех $i$. Для простоты сдвинем начало координат в точку $x_{p}^{M}$, т.е. $x_{p}^{M}=0$ и $s(p, M)=0$.

Легко показать, что найдутся два вектора из набора $\left\{p_{i}\right\}_{i=1}^{k}$ (без ограничения общности это $p_{1}$ и $p_{2}$ ) такие, что

$$
p=\mu_{1} p_{1}+\mu_{2} p_{2}, \quad \mu_{i}>0, \quad i=1,2 .
$$

Фиксируем $q \in \partial B_{1}(0)$. Для $q$ имеется по крайней мере одна из трех возможностей:

1) $q$ лежит в конической оболочке пары векторов $p_{1}$ и $p_{2}$;

2) $q$ лежит в конической оболочке пары векторов $p_{i}$ и $x_{p_{i}}^{M}$, где $i=1,2$;

3) $q$ лежит в конической оболочке векторов $x_{p_{1}}^{M}$ и $x_{p_{2}}^{M}$. 
Рассмотрим каждьй из этих случаев.

Случай 1. Имеем $q=\alpha_{1} p_{1}+\alpha_{2} p_{2}$, где $\alpha_{i}>0$. Пользуясь вьпуклостью и положительной однородностью опорной функции, получаем

$$
\begin{aligned}
s\left(q, \bigcap_{i=1}^{k}\left(M-x_{p_{i}}^{M}\right)\right) & \leqslant \sum_{i=1}^{2} \alpha_{i} s\left(p_{i}, \bigcap_{j=1}^{k}\left(M-x_{p_{j}}^{M}\right)\right) \\
& \leqslant \sum_{i=1}^{2} \alpha_{i} s\left(p_{i}, M-x_{p_{i}}^{M}\right)=0 \leqslant s(q, M) .
\end{aligned}
$$

Случай 2. Пусть, для определенности, $i=1$. Тогда $\left\langle p_{1}, x_{p_{1}}^{M}\right\rangle=s\left(p_{1}, M\right) \geqslant 0$, $0 \in M$. Отсюда следует, что угол между векторами $p_{1}$ и $x_{p_{1}}^{M}$ не больше $\pi / 2$. Отсюда, в свою очередь, вытекает, что угол между векторами $q$ и $x_{p_{1}}^{M}$ также не превосходит $\pi / 2$. Следовательно,

$$
s\left(q, \bigcap_{i=1}^{k}\left(M-x_{p_{i}}^{M}\right)\right) \leqslant s(q, M)-\left\langle q, x_{p_{1}}^{M}\right\rangle \leqslant s(q, M) .
$$

Случай 3 . Так как $s(p, M)=0$, то векторы $x_{p_{i}}^{M}$ лежат в полуплоскости $\{x:$ $\langle p, x\rangle \leqslant 0\}$, поэтому угол между $x_{p_{1}}^{M}$ и $x_{p_{2}}^{M}$ не больше $\pi$. Следовательно, угол между $q$ и одним из векторов $x_{p_{i}}^{M}, i=1,2$, не превосходит $\pi / 2$. Пусть, для определенности, угол между $x_{p_{1}}^{M}$ и $q$ не более $\pi / 2$. Имеем

$$
s\left(q, \bigcap_{i=1}^{k}\left(M-x_{p_{i}}^{M}\right)\right) \leqslant s(q, M)-\left\langle q, x_{p_{1}}^{M}\right\rangle \leqslant s(q, M) .
$$

Итак, поскольку для любого $q \in \partial B_{1}(0)$ реализуется один из случаев $1-3$, то

$$
s\left(q, \bigcap_{i=1}^{k}\left(M-x_{p_{i}}^{M}\right)\right) \leqslant s(q, M) \quad \forall q \in \partial B_{1}(0) .
$$

Отсюда вытекает требуемое включение.

Из теоремы 2.5 и леммы 2.2 непосредственно получаем

СлЕДСТВИЕ 2.6. Любой выпуклый компакт из $\mathbb{R}^{2}$ является порождающим множеством.

Лемма 2.3. Пусть $\mathscr{H}$ - гильбертово пространство, L - замкнутое подпространство в $\mathscr{H}$ и $\pi$ - ортогональный проектор $\mathscr{H}$ на L. Тогда если $L \cap B_{R}(x) \neq \varnothing$, mo

$$
L \cap B_{R}(x)=\left\{y \in L:\|y-\pi x\| \leqslant \sqrt{R^{2}-\|x-\pi x\|^{2}}\right\} .
$$


ДокАЗАТЕЛЬСтво. Точка $y \in L \cap B_{R}(x)$ тогда и только тогда, когда $y \in L$ и $\|y-x\|^{2} \leqslant R^{2}$. Это эквивалентно

$$
\|y-\pi x-(x-\pi x)\|^{2} \leqslant R^{2}, \quad\langle y-\pi x, x-\pi x\rangle=0
$$

откуда получаем (2.7).

ТеОрема 2.6. Пусть $\mathscr{H}$ - гильбертово пространство, $R>0$. Тогда шар $B_{R}(0)$ в $\mathscr{H}$ является порождающим множеством.

Доказательство. Пусть $A=\bigcap_{x \in X} B_{R}(x), X \subset \mathscr{H}$. Допустим противное, тогда по теореме 1.1 сушествует такое $p \in \partial B_{1}^{*}(0)$, для которого

$$
z \in A \backslash B_{R}\left(x_{p}-p R\right), \text { где } x_{p} \in A, \quad\left\langle p, x_{p}\right\rangle=s(p, A) .
$$

Пусть $L=\operatorname{aff}\left\{x_{p}, z, x_{p}-p R\right\}$. Покажем, что $L-$ двумерное линейное многообразие в $\mathscr{H}$. Действительно, $z \notin\left\{x_{p}+\lambda p: \lambda>0\right\},\langle p, z\rangle \leqslant\left\langle p, x_{p}\right\rangle=s(p, A)$; $z \notin\left\{x_{p}-2 p R-\lambda p: \lambda>0\right\}$, иначе $\left\|x_{p}-z\right\|>2 R$, что противоречит определению $A$; $z \notin\left\{x_{p}-\lambda p: \lambda \in[0,2 R]\right\}$, так как $\left\|z-\left(x_{p}-p R\right)\right\|>R$. Итак, $z$ не лежит на прямой aff $\left\{x_{p}, x_{p}-p R\right\}$, т.е. $L$ двумерно.

Без ограничения общности будем считать, что $x_{p}-p R=0$ и $L=\operatorname{lin}\left\{e_{1}, e_{2}\right\}$, где $\left\{e_{1}, e_{2}\right\}$ - ортонормированный базис в $L$. Пусть $\pi$ - ортогональный проектор на $L$.

Подпространство $L$ с базисом $\left\{e_{1}, e_{2}\right\}$ изоморфно $\mathbb{R}^{2}$. По лемме 2.3

$$
\begin{aligned}
& B_{L}=B_{R}(0) \cap L=\left\{\left(\xi_{1}, \xi_{2}\right) \in L: \xi_{1}^{2}+\xi_{2}^{2} \leqslant R^{2}\right\}, \\
& A_{L}=A \cap L=\bigcap_{x \in X}\left\{\left(\xi_{1}, \xi_{2}\right) \in L:\left(\xi_{1}-\pi_{1}(x)\right)^{2}+\left(\xi_{2}-\pi_{2}(x)\right)^{2} \leqslant r_{x}^{2}\right\},
\end{aligned}
$$

где $\left(\pi_{1}(x), \pi_{2}(x)\right)=\pi x$, а $r_{x}=\sqrt{R^{2}-\|x-\pi x\|^{2}}$. Отметим, что $z \in A_{L} \backslash B_{L}$.

Так как $x_{p} \in A_{L}$, то $\left\langle p, x_{p}\right\rangle \leqslant s\left(p, A_{L}\right) \leqslant s(p, A)=\left\langle p, x_{p}\right\rangle$, т.е. $x_{p} \in A_{L}(p)$. Аналогично $x_{p} \in B_{L}(p)$.

Итак, в базисе $\left\{e_{1}, e_{2}\right\} B_{L}$ изоморфно кругу радиуса $R$ в $\mathbb{R}^{2}, A_{L}$ изоморфно пересечению кругов радиусов не более $R, x_{p}$-опорньй элемент $A_{L}$ и $B_{L}$ в направлении $p$. По теоремам 2.6 и 1.1 это влечет $A_{L} \subset B_{L}$. Но это противоречит тому, что $z \in A_{L} \backslash B_{L}$. Противоречие показывает, что $B_{R}(0)$ - порождаюшее множество.

\section{§ 3. Надграфик параболы - порождающее множество}

Покажем, что надграфик параболы является порождающим множеством в пространстве $\mathscr{H} \times \mathbb{R}$, где $\mathscr{H}$ - гильбертово пространство. При этом изучим некоторые свойства сильно выпуклых функций. Следуя [14], напомним определение сильно вьпуклой функции.

ОПредЕЛЕниЕ 3.1. Функция $f: \mathscr{H} \rightarrow \overline{\mathbb{R}}$ называется сильно выпуклой с константой $\varkappa>0$, если сушествует собственная полунепрерьвная снизу выпуклая функция $g: \mathscr{H} \rightarrow \overline{\mathbb{R}}$ такая, что $f(x)=g(x)+\frac{1}{2} \varkappa\|x\|^{2}, x \in \mathscr{H}$. 


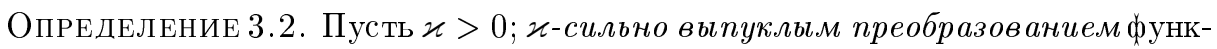
ции $f: \mathscr{H} \rightarrow \overline{\mathbb{R}}$ назовем функцию $f_{\varkappa}^{\#}: \mathscr{H} \rightarrow \overline{\mathbb{R}}$ вида

$$
f_{\varkappa}^{\#}\left(x^{*}\right)=\sup _{x \in \mathscr{H}}\left(\frac{\varkappa}{2}\left\|x+x^{*}\right\|^{2}-f(x)\right) .
$$

Из определений 3.1 и 3.2 следует, что

$$
f_{\varkappa}^{\#}\left(x^{*}\right)=\frac{\varkappa}{2}\left\|x^{*}\right\|^{2}+g^{*}\left(\varkappa x^{*}\right)
$$

где $g^{*}\left(x^{*}\right)$ есть преобразование Юнга-Фенхеля (см., например, [16], [17]) функции $g(x)=f(x)-\frac{1}{2} \varkappa\|x\|^{2}$.

Применяя (3.1) к функции $f_{\varkappa}^{\#}\left(x^{*}\right)$, получаем второе $\varkappa$-сильно выпуклое преобразование $f_{\varkappa \varkappa}^{\# \#}(x)$. Используя формулу $(3.2)$ и свойства преобразования Юнга-Фенхеля [16], [17], получаем, что если $f$ есть собственная сильно выпуклая функция с константой $\varkappa>0$, то $f_{\varkappa}^{\#}\left(x^{*}\right)$ является собственной полунепрерывной снизу сильно выпуклой функцией с той же константой $\varkappa$, при этом справедливы неравенства:

$$
\begin{gathered}
f(x)+f_{\varkappa}^{\#}\left(x^{*}\right) \geqslant \frac{\varkappa}{2}\left\|x+x^{*}\right\|^{2} \quad \forall x, x^{*} \in \mathscr{H}, \\
f_{\varkappa \varkappa}^{\# \#}(x) \leqslant f(x), \quad x \in \mathscr{H} .
\end{gathered}
$$

ПреДЛОЖЕнИЕ 3.1. Пусть $f: \mathscr{H} \rightarrow \overline{\mathbb{R}}$ - собственная функция. Тогда $f$ является полунепрерывной снизу сильно выпуклой функцией с константой $\varkappa>0$ тогда и только тогда, когда справедливо равенство

$$
f_{\varkappa \varkappa}^{\# \#}=f
$$

ДокаЗАТЕльСтво. Если верно равенство (3.5), то $f$ сильно выпукла и полунепрерывна снизу. Если $f$ - сильно выпуклая функция, то в силу определения 3.1 получим

$$
f_{\varkappa \varkappa}^{\# \#}(x)=\frac{\varkappa}{2}\|x\|^{2}+g^{* *}(x),
$$

где $g^{* *}$ - вторая сопряженная Юнга-Фенхеля функции $g$. Так как $g-$ собственная полунепрерывная снизу выпуклая функция, то по теореме Фенхеля-Моро справедливо равенство $g=g^{* *}$, откуда следует равенство (3.5).

СЛЕДСТВИЕ 3.1. Всякая собственная полунепрерывная снизу сильно выпуклая функция $f: \mathscr{H} \rightarrow \overline{\mathbb{R}}$ с константой $\varkappa>0$ совпадает с верхней гранью семейства всех не превосходящих ее парабол вида $\varphi_{a, b}(x)=\frac{1}{2} \varkappa\|x+a\|^{2}+b$.

ДокАЗАТЕЛЬСтво. В самом деле, в силу (3.5) получаем равенство

$$
f(x)=\sup _{x^{*} \in \operatorname{dom} f_{\varkappa}^{\#}}\left(\frac{\varkappa}{2}\left\|x+x^{*}\right\|^{2}-f_{\varkappa}^{\#}\left(x^{*}\right)\right) .
$$




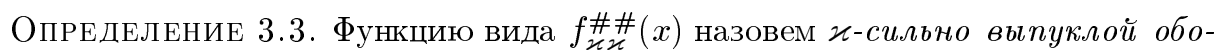
лочкой функции $f: \mathscr{H} \rightarrow \mathbb{R}$, т.е.

$$
\operatorname{strco}_{\varkappa} f(x)=\frac{\varkappa}{2}\|x\|^{2}+\left(f(x)-\frac{\varkappa}{2}\|x\|^{2}\right)^{* *} .
$$

ТеОрема 3.1. Пусть $\varkappa>0$. Тогда в пространстве $\mathscr{H} \times \mathbb{R}$ надграфик парабольи $M_{\varkappa}=\left\{(x, y) \in \mathscr{H} \times \mathbb{R}: y \geqslant \frac{1}{2} \varkappa\|x\|^{2}\right\}$ является порожсдающим множеством. При этом надграфики сильно выпукльх с данной константой $\varkappa$ функций и только они являются $M_{\varkappa}$-сильно выпукльми множсествами.

ДокАЗАТЕльство. Для всякой сильно выпуклой функции $f$ с данной константой $\varkappa$ в силу следствия 3.1 получаем равенство

$$
\text { epi } f=\bigcap_{x^{*} \in X} \operatorname{epi} \varphi_{x^{*}, \alpha},
$$

где $\varphi_{x^{*}, \alpha}(x)=\frac{1}{2} \varkappa\left\|x+x^{*}\right\|^{2}-\alpha, \alpha=f_{\varkappa}^{\#}\left(x^{*}\right), X=\operatorname{dom} f_{\varkappa}^{\#}$.

Справедливо и обратное. Если для функции $f \neq+\infty$ верно равенство (3.8), где $\varphi_{x^{*}, \alpha}(x)$ - некоторое семейство парабол с коэффициентом $\varkappa$, то

$$
\begin{aligned}
f(x) & =\sup _{\left(x^{*}, \alpha\right) \in Y}\left(\frac{\varkappa}{2}\left\|x+x^{*}\right\|^{2}-\alpha\right) \\
& =\frac{\varkappa}{2}\|x\|^{2}+\sup _{\left(x^{*}, \alpha\right) \in Y}\left(\left\langle\varkappa x, x^{*}\right\rangle-\left(\alpha-\frac{\varkappa}{2}\left\|x^{*}\right\|^{2}\right)\right)=\frac{\varkappa}{2}\|x\|^{2}+h^{*}(\varkappa x),
\end{aligned}
$$

где $h^{*}$ есть собственная (по условию) функция, причем она вьпукла и полунепрерывна снизу как сопряженная Юнга-Фенхеля функция к некоторой функции $h$, легко определяемой множеством $Y$. В итоге показали, что $f$ есть собственная полунепрерывная снизу сильно выпуклая функция.

Поэтому чтобы доказать, что $M_{\varkappa}$ является порождаюшим множеством в $\mathscr{H} \times \mathbb{R}$, достаточно проверить, что для любой собственной полунепрерывной снизу сильно вьпуклой функции $f$ найдется функция $h$ такая, что

$$
\text { epi } f+\text { epi } h=\text { epi }\left(\frac{\varkappa}{2}\|\cdot\|^{2}\right) \text {. }
$$

Покажем, что (3.9) верно при $h=f_{\varkappa}^{\#}$. Легко проверить, что при этом равенство (3.9) эквивалентно равенству функций

$$
\inf _{y \in \mathscr{H}}\left\{f(x-y)+f_{\varkappa}^{\#}(y)\right\}=\frac{\varkappa}{2}\|x\|^{2} \quad \forall x \in \mathscr{H} .
$$

В силу неравенства (3.3) достаточно доказать, что для любого $x \in \mathscr{H}$ найдутся $x_{1}$ и $x_{2}$ из $\mathscr{H}$ такие, что

$$
f\left(x_{1}\right)+f_{\varkappa}^{\#}\left(x_{2}\right)=\frac{\varkappa}{2}\|x\|^{2}, \quad x_{1}+x_{2}=x .
$$

Ввиду определений 3.1 и 3.2 равенство (3.11) эквивалентно равенству

$$
g\left(x_{1}\right)+g^{*}\left(\tilde{x}_{2}\right)=\left\langle x_{1}, \tilde{x}_{2}\right\rangle,
$$

где $\tilde{x}_{2}=\varkappa x_{2}$, т.е. $x_{1}+\varkappa^{-1} x_{2}=x$.

В силу [16; $§ 4.2$, предложение 1] равенство (3.12) эквивалентно включению $\tilde{x}_{2} \in \partial g\left(x_{1}\right)$. В итоге, (3.9) эквивалентно тому, что для всякой собственной полунепрерывной снизу выпуклой функции $g: \mathscr{H} \rightarrow \mathbb{R}$ для любого $x \in \mathscr{H}$ и для любого $\varkappa>0$ найдется $x_{1} \in \mathscr{H}$ такой, что $x \in x_{1}+\varkappa^{-1} \partial g\left(x_{1}\right)$. Последнее утверждение доказано в [15; теорема 4.3.11]. 


\section{§4. Простейшие свойства $M$-сильно выпуклых множеств}

ПРЕДЛОЖЕНИЕ 4.1.

1. Пусть $A \subset E$ есть $M_{1}$-сильно выпуклое множество, а $M_{1}$ является $M_{2}$-сильно выпуклым множеством. Тогда А будет $M_{2}$-сильно выпуклым множсеством.

2. Пусть $A_{1}, A_{2} \subset E$, причем $A_{1}$ является $M$-сильно выпукльм. Тогда если мнохсество $A_{1} \stackrel{*}{*} A_{2}$ непусто, то $M$-сильно выпукло.

3. Пусть дань два порождающие множсества $M_{1}$ и $M_{2}$ такие, что множество $M=M_{1}+M_{2}$ замкнуто, и два множества $A_{1}$ и $A_{2}$, являющиеся соответственно $M_{1}-$ и $M_{2}$-сильно выпуклыми. Тогда для множества $A=A_{1}+A_{2}$ найдется выпуклое замкнутое множество $B$ такое, что справедливо равенство $A+B=M$.

4. Выпуклое замкнутое множество $А$ является $M$-сильно выпукльмм тогда и только тогда, когда разность опорных функиий $s(p, M)-$ $s(p, A)$ есть выпуклая собственная полунепрерывная снизу функиия.

5. Пусть $f: E^{*} \rightarrow \mathbb{R}$ - собственная положительно однородная полунепрерьвная снизу функиия такая, что $\overline{\mathrm{co}} f>-\infty$, a $M \subset E$ есть порожсдающее множество. Пусть разность опорных функиий $s(p, M)-$ $f(p)$ является собственной выпуклой и полунепрерывной снизу функиией, тогда и разность $s(p, M)-\overline{\mathrm{co}} f(p)$ также является собственной выпуклой и полунепрерывной снизу функиией.

6. Пусть дана последовательность ограниченных порожсдающих множеств $M_{k}$, для которой существует выпуклое замкнутое ограниченное множество $M$ такое, что для всех $k$ справедливь равенства $M_{k}+\left(M * M_{k}\right)=M$. Пусть $\left\{A_{k}\right\}-$ последовательность $M_{k}$-сильно выпукльх множсеств, сходящаяся $\kappa A$ в метрике Хаусдорфа. Тогда существует выпуклое замкнутое мнохсество $B$ такое, что справедливо равенство $A+B=M$.

ДокАЗАТЕльство. Справедливость пп. 1 и 2 легко следует из определений 1.1 и $1.2 ;$ п. 4 вытекает из теоремы 1.1 .

3. Имеем $A_{i}+B_{i}=M_{i}, i=1,2$. Складывая равенства, получаем, что $A+$ $\left(B_{1}+B_{2}\right)=M$.

5. Из условия следует, что найдется выпуклое замкнутое множество $X$ из $E$ такое, что $s(p, X)=s(p, M)-f(p)$ (см. [20; гл. 1]). Так как $\overline{\operatorname{co}} f \leqslant f$ и $\overline{\operatorname{co}} f>-\infty$, то со $f$ есть опорная функция некоторого множества $Y \subset E$, т.е. $\overline{\operatorname{co}} f(p)=s(p, Y)$. Итак,

$$
s(p, Y)=\overline{\mathrm{co}}(s(p, M)-s(p, X)),
$$

т.е. $Y=M \stackrel{*}{X}$ является $M$-сильно выпуклым множеством, причем

$$
s(p, M)-\overline{\operatorname{co}} f(p)=s(p, M)-s(p, Y)
$$

следовательно, по п. 4 последняя функция является собственной выпуклой и полунепрерывной снизу.

6. Для любого $k$ найдется выпуклое множество $B_{k}$ такое, что $A_{k}+B_{k}=M_{k}$. Отсюда $A_{k}+B_{k}+\left(M \stackrel{*}{*} M_{k}\right)=M$. Определим $C_{k}=B_{k}+\left(M \stackrel{*}{*} M_{k}\right)$ для всех $k$. 
Имеем $A_{k}+C_{k}=M$ для всех $k$. Из сходимости $A_{k}$ к $A$ в метрике Хаусдорфа следует сходимость $C_{k}$ к $C$ в метрике Хаусдорфа, причем $A+C=M$ по лемме 1.1.

ТЕОРема 4.1. Пусть $R>0$ и ограниченное выпуклое замкнутое множество $A$ является $B_{R}(0)$-сильно выпуклым множеством в гильбертовом пространстве Н̈. Тогда градиент опорной функиии множества А существует и удовлетворяет условию Липшица на $\partial B_{1}(0)$ с константой $R$, m.e.

$$
\left\|x_{p}^{A}-x_{q}^{A}\right\| \leqslant R\|p-q\| \quad \forall p, q \in \partial B_{1}(0)
$$

ДоКАЗАТЕльство. Для любого $p \in \partial B_{1}(0)$ по лемме 1.2 опорное множество $A(p)$ непусто. Из определений субдифференциала и опорной функции следует, что $A(p)=\partial s(p, A) \forall p \in \partial B_{1}(0)$. Так как в силу строгой выпуклости $A$ множество $A(p)$ состоит из одной точки $x_{p}^{A}$, то это означает сушествование градиента опорной функции. Таким образом, остается доказать неравенство (4.1). Отметим, что для $M=B_{R}(0)$ каждое множество $M(p)$, где $p \in \partial B_{1}(0)$, очевидно, состоит из одной точки $p R$. По опорному принципу (см. следствие 1.1$)$ для любых $p, q \in \partial B_{1}(0)$ получаем $x_{p}^{A} \in A \subset B_{R}\left(x_{q}^{A}-q R\right), x_{q}^{A} \in A \subset B_{R}\left(x_{p}^{A}-p R\right)$. Отсюда следует, что

$$
\left\|x_{p}^{A}-x_{q}^{A}\right\|^{2} \leqslant 2 R\left\langle q, x_{q}^{A}-x_{p}^{A}\right\rangle, \quad\left\|x_{p}^{A}-x_{q}^{A}\right\|^{2} \leqslant 2 R\left\langle p, x_{p}^{A}-x_{q}^{A}\right\rangle .
$$

Складывая два последних неравенства, получаем, что $2\left\|x_{p}^{A}-x_{q}^{A}\right\|^{2} \leqslant 2 R\langle p-q$, $\left.x_{p}^{A}-x_{q}^{A}\right\rangle$, откуда следует неравенство (4.1).

\section{§5. $M$-сильно выпуклая оболочка множеств}

Введем понятие $M$-сильно выпуклой оболочки множества, обобщающее классическое понятие выпуклой оболочки множества и понятие $r$-сильно выпуклой оболочки множества, рассмотренной в работе [9].

ОПРЕДЕЛЕНИЕ 5.1. Пусть $M \subset E$ - выпуклое замкнутое множество. Для всякого множества $A$ такого, что $M \stackrel{*}{A} \neq \varnothing, M$-сильно выпуклой оболочкой множества $A$ назовем множество вида

$$
\operatorname{strco}_{M} A=M \stackrel{*}{ }(M \stackrel{*}{*} A) .
$$

Очевидно, что опорная функция $M$-сильно вьпуклой оболочки множества $A$ имеет вид

$$
s\left(p, \operatorname{strco}_{M} A\right)=\overline{\mathrm{co}}(s(p, M)-\overline{\mathrm{co}}(s(p, M)-s(p, A))) .
$$

ЗАмЕчАниЕ 5.1. В случае, когда множество $M$ ограничено, операцию замыкания выпуклых оболочек в формуле (5.1) можно опустить.

ЗАмЕчАнИЕ 5.2. Операция взятия $M$-сильно выпуклой оболочки множества наследует многие полезные в приложениях свойства $r$-сильно выпуклой оболочки, описанные в [9]. 
Теорема 5.1. Пусть $M-$ порохсдающее множество и $A$ - такое множсество из $E$, что $M \stackrel{*}{*} \neq \varnothing$. Тогда множество $\operatorname{strco}_{M} A$ является наименьиим по включению $M$-сильно выпукльм множсеством, содержащим $A$. Опорная функция әтого множсества задается формулой

$$
\begin{gathered}
s\left(p, \operatorname{strco}_{M} A\right)=s(p, M)-\overline{\operatorname{co}}(s(p, M)-s(p, A)), \quad p \in b(M) \\
s\left(p, \operatorname{strco}_{M} A\right)=+\infty, \quad p \notin b(M) .
\end{gathered}
$$

ДокАЗАТЕЛьство. Из включения $A+(M * A) \subset M$, которое справедливо всегда (см., например, [19; гл. 1, $\S 1$, формула (3)]), имеем $A \subset \operatorname{strco}_{M} A$. Так как $\operatorname{strco}_{M} A=M \stackrel{*}{X}$, где $X=M \stackrel{*}{A}$, то $\operatorname{strco}_{M} A$ является $M$-сильно выпукльп множеством.

Пусть дано произвольное $M$-сильно выпуклое множество $C$, содержащее $A$. Имеем $M$ * $C \subset M \stackrel{*}{A}$, отсюда в силу того, что $C$ есть $M$-сильно вьпуклое множество, получаем $C=M *(M * C) \supset \operatorname{strco}_{M} A$. Итак, $\operatorname{strco}_{M} A$ есть наименьшее по включению $M$-сильно выпуклое множество, содержашее $A$.

В силу определения порождаюшего множества $M \operatorname{strco}_{M} A+(M \stackrel{*}{A})=M$. Отсюда следует формула для опорной функции $\operatorname{strco}_{M} A$.

Приведем некоторые свойства $M$-сильно вьпуклой оболочки множеств.

TEOPEMA 5.2 .

1. Пусть $A_{1} \subset A_{2} u M \stackrel{*}{*} A_{2} \neq \varnothing$. Tогдa $\operatorname{strco}_{M} A_{1} \subset \operatorname{strco}_{M} A_{2}$.

2. Пусть $M \stackrel{*}{*} A \neq \varnothing$. Тогда для любого $\alpha>0$ имеем $\operatorname{strco}_{\alpha M}(\alpha A)=$ $\alpha \operatorname{strco}_{M} A$.

3. Пусть выпуклье замкнутые множсества $M_{1}$ и $M_{2}$ таковы, что $M_{1}+$ $\left(M_{2} * M_{1}\right)=M_{2}$. Пусть $A-$ такое многество, что $M_{1} * A \neq \varnothing$. Тогда $\overline{\mathrm{co}} A \subset \operatorname{strco}_{M_{2}} A \subset \operatorname{strco}_{M_{1}} A$.

4. Пусть $M_{1}$ и $M_{2}$ - порождающие множества, причем $M_{1}+M_{2}-$ замкнутое множество. Пусть $A_{1}$ и $A_{2}$ - такие множества, что $M_{i} * A_{i} \neq \varnothing$, $i=1,2$. Тогда $\operatorname{strco}_{M_{1}}+M_{2}\left(A_{1}+A_{2}\right) \subset \operatorname{strco}_{M_{1}} A_{1}+\operatorname{strco}_{M_{2}} A_{2}$. Ecли $A_{2}=M_{2}$, то в последнем включении имеет место равенство.

5. Пусть $M$ - выпуклое замкнутое множество, $A$ и $B$ - такие множесmва, что $M \stackrel{*}{*} A \neq \varnothing u A \stackrel{*}{ } B \neq \varnothing$. Tогда $\operatorname{strco}_{M}(A \stackrel{*}{*} B) \subset \operatorname{strco}_{M} A * *$ $B$.

ДоКАЗАТЕЛЬСтво. Пункты 1 и 2 очевидно следуют из определения 5.1.

3. Первое включение вытекает из определения 5.1 и из того, что $M_{2}$ * $A=$ $M_{2} * \overline{\operatorname{co}} A$ и $A \subset \operatorname{strco}_{M_{2}} A$.

По определению 5.1 имеем со $M_{2} A+M_{2} * A=M_{2}$, по условию теоремы

$$
M_{2} \stackrel{*}{A} A=\left(M_{1}+\left(M_{2} \stackrel{*}{*} M_{1}\right)\right) \stackrel{*}{A} \supset M_{1} \stackrel{*}{A} A+M_{2} \stackrel{*}{*} M_{1} .
$$

Поэтому

$$
\operatorname{strco}_{M_{2}} A+M_{1} \stackrel{*}{*} A+M_{2} \stackrel{*}{*} M_{1} \subset M_{1}+M_{2} \stackrel{*}{*} M_{1},
$$

откуда

$$
\operatorname{strco}_{M_{2}} A+M_{1} \stackrel{*}{*} A \subset M_{1}=\operatorname{strco}_{M_{1}} A+M_{1} \stackrel{*}{ } A,
$$

следовательно, $\operatorname{strco}_{M_{2}} A \subset \operatorname{strco}_{M_{1}} A$. 
4. По определению 5.1 имеем

$$
\begin{aligned}
\operatorname{strco}_{M_{1}+M_{2}}\left(A_{1}+A_{2}\right) & =\left(M_{1}+M_{2}\right) \stackrel{*}{ }\left(\left(M_{1}+M_{2}\right) *\left(A_{1}+A_{2}\right)\right) \\
& \subset\left(M_{1}+M_{2}\right) *\left(\left(M_{1} * A_{1}\right)+\left(M_{2} * A_{2}\right)\right) \\
& =\left(\left(M_{1}+M_{2}\right) *\left(M_{1} * A_{1}\right)\right) \stackrel{*}{*}\left(M_{2} * A_{2}\right) \\
& =\left(M_{1} * A_{1}+\operatorname{strco}_{M_{1}} A_{1}+M_{2}\right) \stackrel{*}{*}\left(M_{1} * A_{1}\right) *\left(M_{2} * A_{2}\right) \\
& =\left(\operatorname{strco}_{M_{1}} A_{1}+M_{2}\right) \stackrel{*}{*}\left(M_{2} * A_{2}\right)=\operatorname{strco}_{M_{1}} A+\operatorname{strco}_{M_{2}} A .
\end{aligned}
$$

Если $A_{2}=M_{2}$, то

$$
\begin{aligned}
\operatorname{strco}_{M_{1}+M_{2}}\left(A_{1}+M_{2}\right) & =\left(M_{1}+M_{2}\right) \stackrel{*}{ }\left(\left(\left(M_{1}+M_{2}\right) \stackrel{*}{*} M_{2}\right) \stackrel{*}{*} A_{1}\right) \\
& =M_{2}+\left(M_{1} *\left(M_{1} * A_{1}\right)\right)=\operatorname{strco}_{M_{2}} M_{2}+\operatorname{strco}_{M_{1}} A_{1} .
\end{aligned}
$$

5. Воспользовавшись очевидно верным для любых множеств $M, A, B$ соотношением $M \stackrel{*}{*}(A \stackrel{*}{B}) \supset M \stackrel{*}{ } A+B$, получим

$$
\operatorname{strco}_{M}(A \stackrel{*}{*}) \subset M \stackrel{*}{*}((M \stackrel{*}{*})+B)=\operatorname{strco}_{M} A \stackrel{*}{*} .
$$

ТеОрема 5.3. Пусть $M \subset E$ - ограниченное порохсдающее множсество, $A_{1}$ и $A_{2}$ - множсества из $E$. Пусть существуют $\alpha=\alpha\left(M, A_{1}, A_{2}\right)>0 u$ $x_{i} \in E$ такие, что $B_{\alpha}\left(x_{i}\right) \subset M \stackrel{*}{*} A_{i}, i=1,2$. Тогда

$$
h\left(\operatorname{strco}_{M} A_{1}, \operatorname{strco}_{M} A_{2}\right) \leqslant \frac{\operatorname{diam} M+\alpha}{\alpha} h\left(A_{1}, A_{2}\right) .
$$

ДокаЗАТЕльство. 1. Пусть $h=h\left(A_{1}, A_{2}\right) \leqslant \alpha$. По формуле представления расстояния по Хаусдорфу через опорные функции и по теореме 5.1 получаем

$$
\begin{aligned}
h\left(\operatorname{strco}_{M} A_{1}, \operatorname{strco}_{M} A_{2}\right) & =\sup _{\|p\|_{*}=1}\left|s(p, M)-s\left(p, M * A_{1}\right)-s(p, M)+s\left(p, M * A_{2}\right)\right| \\
& =h\left(M \stackrel{*}{*} A_{1}, M \stackrel{*}{*} A_{2}\right) .
\end{aligned}
$$

Обозначим $\operatorname{diam} M$ через $D(M),(D(M)-\alpha) h / \alpha$ через $k, M * A_{i}$ через $D_{i}, i=1,2$, гранищу $D_{i}$ через $\partial D_{i}$.

Фиксируем $x \in \partial D_{i}$. Рассмотрим $\operatorname{co}\left(\{x\} \cup B_{\alpha}\left(x_{i}\right)\right) \subset D_{i}$. Выберем на отрезке $\left[x_{i}, x\right]$ точку $g$ так, чтобы $\|x-g\| /\left\|x-x_{i}\right\|=h / \alpha$. В силу соображений подобия

$$
g+B_{h}(0) \subset \operatorname{co}\left(\{x\} \cup B_{\alpha}\left(x_{i}\right)\right) \subset D_{i},
$$

откуда $\varrho\left(x, D_{i}\right.$ * $\left.B_{h}(0)\right) \leqslant\|x-g\| \leqslant(D(M)-\alpha) h / \alpha \leqslant k$. В итоге,

$$
h\left(D_{i}, D_{i} * B_{h}(0)\right)=\sup _{x \in \partial D_{i}} \varrho\left(x, D_{i} * B_{h}(0)\right) \leqslant k .
$$

Так как из включения $A_{2} \subset A_{1}+B_{h}(0)$ следует $D_{1} \stackrel{*}{*} B_{h}(0) \subset D_{2}$, то, используя (5.6), получаем

$$
D_{1} \subset D_{1} \stackrel{*}{ } B_{h}(0)+k B_{1}(0) \subset D_{2}+k B_{1}(0)
$$


Очевидно, что аналогичное включение будет справедливо при перестановке $A_{1}$ и $A_{2}$. Отсюда получаем, что $h\left(D_{1}, D_{2}\right) \leqslant k$. В силу (5.5) это влечет (5.4).

2. Пусть $h=h\left(A_{1}, A_{2}\right)>\alpha$. По теореме 5.2 для любого множества $A$ такого, что $M * * A \neq 0$, справедливо включение $\overline{\operatorname{co}} A \subset \operatorname{strco}_{M} A$, т.е. $h\left(\overline{\mathrm{co}} A, \operatorname{strco}_{M} A\right) \leqslant$ $\operatorname{diam}\left(\operatorname{strco}_{M} A\right) \leqslant D(M)$. Отсюда, используя известное свойство липшицевости операции взятия выпуклой оболочки ограниченных множеств (см., например, [21; гл. $5, \S 2$, лемма 4]), получаем

$$
\begin{aligned}
\operatorname{strco}_{M} A_{1} & \subset \overline{\operatorname{co}} A_{1}+D(M) B_{1}(0) \subset \overline{\operatorname{co}} A_{2}+D(M) B_{1}(0)+h B_{1}(0) \\
& \subset \operatorname{strco}_{M} A_{2}+(D(M)+h) B_{1}(0) \\
& \subset \operatorname{strco}_{M} A_{2}+h\left(A_{1}, A_{2}\right)\left(\frac{D(M)}{\alpha}+1\right) B_{1}(0)
\end{aligned}
$$

Аналогичное включение будет также справедливо при перестановке $A_{1}$ и $A_{2}$, откуда и следует оценка (5.4).

ЗАмЕчАниЕ 5.3. У оператора $M$-сильно выпуклой оболочки константа Липшица больше единицы по существу, в отличие от оператора выпуклой оболочки, у которого, как известно, константа Липшица равна единище. Этот факт проиллюстрирован в примере 9.7 .

ЗАмечАниЕ 5.4. В случае, когда порождаюшее множество $M$ является шаром $B_{R}(0) \subset \mathbb{R}^{n}$, константу Липшица операции $R$-сильного овыпукления $\operatorname{strco}_{R}$ удается уточнить. Как показано в работах [9] и [13], если числа $r, R, 0<r<R$, и компакты $A_{1}, A_{2}$ таковы, что $B_{r}(0) \stackrel{*}{*} A_{k} \neq \varnothing, k=1,2$, то справедлива оценка

$$
h\left(\operatorname{strco}_{R} A_{1}, \operatorname{strco}_{R} A_{2}\right) \leqslant L(R, r) h\left(A_{1}, A_{2}\right)
$$

где константа Липшица $L(R, r)$ имеет вид

$$
L(R, r)=\max \left\{\sqrt{\frac{R+r}{R-r}}, 1+\frac{r^{2}}{R(R-r)}\right\} .
$$

Следуя [9], обозначим через $\operatorname{strco}_{R} A$ множество $\operatorname{strco}_{B_{R}}(0) A$.

ТЕОРемА 5.4. Пусть в гильбертовом пространстве $\mathscr{H}$ дано выпуклое замкнутое множество $A$, содержащееся в некотором иаре $B_{r}($ a). Пусть $R>r>0$. Тогда справедлива оценка

$$
h\left(A, \operatorname{strco}_{R} A\right) \leqslant \frac{r^{2}}{R}
$$

ДоказАтельство. Очевидно включение $A \subset \operatorname{strco}_{R} A$. Фиксируем произвольную граничную точку $x \in \partial\left(\operatorname{strco}_{R} A\right), x \notin A$. Обозначим через $P_{A}$ оператор проектирования на выпуклое замкнутое множество $A$. Пусть $P_{A} x=y$. Без ограничения общности полагаем, что $y=0$.

Пусть $p=x /\|x\|, H_{p}^{-}=\{z \in \mathscr{H}:\langle p, z\rangle \leqslant 0\}, H_{p}^{\circ}=\{z \in \mathscr{H}:\langle p, z\rangle=0\}$, $H_{p}^{+}=\{z \in \mathscr{H}:\langle p, z\rangle \geqslant 0\}$. Легко видеть, что $A \subset B_{r}(a) \cap H_{p}^{-}$. По лемме 2.3 
$B_{r}(a) \cap H_{p}^{\circ}$ есть шар в подпространстве $H_{p}^{\circ}$ радиуса $\rho=\sqrt{r^{2}-|\langle p, a\rangle|^{2}}$ с центром в точке $b=P_{H_{p}^{\circ}} a$. Пусть $S=B_{r}(a) \cap B_{R}\left(b-p \sqrt{R^{2}-\rho^{2}}\right)$. Отметим, что $H_{p}^{\circ} \cap$ $B_{R}\left(b-p \sqrt{R^{2}-\rho^{2}}\right)=H_{p}^{\circ} \cap B_{r}(a)$.

Покажем, что $A \subset S$. Для этого достаточно доказать, что $B_{r}(a) \cap H_{p}^{-} \subset S$. Фиксируем $z \in B_{r}(a) \cap H_{p}^{-}$. Последнее означает, что $\langle p, z\rangle \leqslant 0$ и $\|z-a\| \leqslant r$. Легко видеть, что $a=b \pm p \sqrt{r^{2}-\rho^{2}}$. Пусть, для определенности, $a=b-p \sqrt{r^{2}-\rho^{2}}$ (другой случай рассматривается аналогично). Подставляя значение $a$, получаем, что $\left\|z-b+p \sqrt{r^{2}-\rho^{2}}\right\| \leqslant r$.

Возводя последнее неравенство в квадрат и учитывая, что $\langle p, b\rangle=0$, получаем $\|z-b\|^{2}+2 \sqrt{r^{2}-\rho^{2}}\langle p, z\rangle \leqslant \rho^{2}$. Так как $\langle p, z\rangle \leqslant 0$, то мы лишш усилим последнее неравенство, заменив коэффициент $\sqrt{r^{2}-\rho^{2}}$ на больший $\sqrt{R^{2}-\rho^{2}}$. В итоге получим $\|z-b\|^{2}+2 \sqrt{R^{2}-\rho^{2}}\langle p, z\rangle \leqslant \rho^{2}$, откуда следует $z \in S$.

Итак, $A \subset S$, следовательно, и $\operatorname{strco}_{R} A \subset S$, так как $S$ является $B_{R}(0)$-сильно вьпуклым множеством.

Убедимся в том, что

$$
S \cap H_{p}^{+}=H_{p}^{+} \cap B_{R}\left(b-p \sqrt{R^{2}-\rho^{2}}\right) .
$$

Для этого достаточно показать, что

$$
H_{p}^{+} \cap B_{r}(a) \supset H_{p}^{+} \cap B_{R}\left(b-p \sqrt{R^{2}-\rho^{2}}\right) .
$$

Как и вьше, считаем, что $a=b-p \sqrt{R^{2}-\rho^{2}}$ (случай $a=b+p \sqrt{R^{2}-\rho^{2}}$ рассматривается аналогично). Фиксируем $z$ из правой части последнего включения. Это значит, что $\left\|z-b+p \sqrt{R^{2}-\rho^{2}}\right\| \leqslant R$, т.е. $\|z-b\|^{2}+2 \sqrt{R^{2}-\rho^{2}}\langle p, z\rangle \leqslant \rho^{2}$, откуда, учитывая, что $\langle p, z\rangle \geqslant 0$, получаем $\|z-b\|^{2}+2 \sqrt{r^{2}-\rho^{2}}\langle p, z\rangle \leqslant \rho^{2}$, т.е. $z \in B_{r}\left(b-p \sqrt{r^{2}-\rho^{2}}\right)=B_{r}(a)$.

Из включения $\operatorname{strco}_{R} A \subset S$ и формулы (5.9) получаем, что

$$
\varrho(x, A)=\|x-0\| \leqslant \sup \left\{\varrho\left(z, H_{p}^{\circ}\right): z \in \partial B_{R}\left(b-p \sqrt{R^{2}-\rho^{2}}\right),\langle p, z\rangle \geqslant 0\right\} .
$$

Покажем, что последний супремум, который обозначим через $I$, равен $R^{2}-$ $\sqrt{R^{2}-\rho^{2}}$.

Взяв $z_{0}=b-p \sqrt{R^{2}-\rho^{2}}+p R$, получим

$$
\varrho\left(z_{0}, H_{p}^{\circ}\right)=R-\sqrt{R^{2}-\rho^{2}} \leqslant I .
$$

Выбрав произвольную точку $q \in \partial B_{1}(0)$ и соответствуюшую точку $z=b-$ $p \sqrt{R^{2}-\rho^{2}}+q R$, из условия $\langle p, z\rangle \geqslant 0$ получим, что $\langle p, q\rangle \geqslant \sqrt{R^{2}-\rho^{2}} / R$. Отсюда в силу того, что $\varrho\left(z, H_{p}^{\circ}\right)=|\langle p, z\rangle|$, имеем

$$
|\langle p, z\rangle|=\langle p, z\rangle=\langle p, q\rangle R-\sqrt{R^{2}-\rho^{2}}<R-\sqrt{R^{2}-\rho^{2}} .
$$

Итак, $I=R-\sqrt{R^{2}-\rho^{2}}$, откуда получаем, что $I \leqslant r^{2} / R$. Так как точка $x \in$ $\partial\left(\operatorname{strco}_{R} A\right)$ выбрана произвольно, то

$$
h\left(A, \operatorname{strco}_{R} A\right)=\sup \left\{\varrho(x, A): x \in \partial\left(\operatorname{strco}_{R} A\right)\right\} \leqslant \frac{r^{2}}{R} .
$$




\section{§6. Обобщение теоремы Каратеодори}

Докажем теорему, являющуюся аналогом теоремы Каратеодори (см., например, [16], [17]) в случае, когда вместо обычной вьпуклой оболочки множества из $\mathbb{R}^{n}$ рассматривается $M$-сильно выпуклая оболочка множества, причем выбранное порождаюшее множество $M$ является строго выпуклым компактом из $\mathbb{R}^{n}$.

Tеорема 6.1. Пусть $M \subset \mathbb{R}^{n}$ есть компактное строго выпуклое порождающее множество. Пусть $A$ - компактное подмноэсество из $\mathbb{R}^{n}$ такое, что $M \stackrel{*}{A} \neq \varnothing$. Тогда любая точка множества $\operatorname{strco~}_{M} A$ принадлежит $M$-сильно выпуклой оболочке некоторого подмножества из $A$, состоящего не более чем из $n+1$ точек.

ДоказАТЕЛЬСтво. 1. Предположим, что $\operatorname{int}(M \stackrel{*}{*} A) \neq \varnothing$. Фиксируем произвольную точку $u \in \partial\left(\operatorname{strco}_{M} A\right)$. Так как множество $M$ строго выпукло, то и

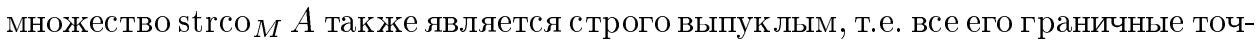
ки являются выступающими.

По определению выступаюшей точки найдется вектор $p \in \partial B_{1}(0)$ такой, что $\langle p, u\rangle=s\left(p, \operatorname{strco}_{M} A\right)$ и

$$
\left\{x \in \mathbb{R}^{n}:\langle p, x\rangle=\langle p, u\rangle\right\} \cap \operatorname{strco}_{M} A=\{u\} .
$$

По теореме 5.1 и по [17; следствие 17.1.6] получаем

$$
s\left(p, \operatorname{strco}_{M} A\right)=s(p, M)-\inf \left\{\sum_{i=1}^{n}\left(s\left(p_{i}, M\right)-s\left(p_{i}, A\right)\right): \sum_{i=1}^{n} p_{i}=p\right\},
$$

а в силу непустоты внутренности множества $M \stackrel{*}{*} A$ по [19; гл. $1, \S 5$, предложение 14] инфинум в операции вычисления выпуклой оболочки разности опорных функций $s(p, M)-s(p, A)$ достигается. Поэтому найдется набор векторов $\left\{p_{i}\right\}_{i=1}^{n} \in \mathbb{R}^{n}$ такой, что $\sum_{i=1}^{n} p_{i}=p$ и

$$
s\left(p, \operatorname{strco}_{M} A\right)=s(p, M)-\sum_{i=1}^{n}\left(s\left(p_{i}, M\right)-s\left(p_{i}, A\right)\right) .
$$

При $p_{i} \neq 0$ выберем $x_{i} \in A$ так, чтобы $\left\langle p_{i}, x_{i}\right\rangle=s\left(p_{i}, A\right)$. При $p_{i}=0$ выберем $x_{i} \in A$ произвольно. Обозначим множество точек $\left\{x_{i}\right\}_{i=1}^{n} \subset A$ через $S$. Тогда $\operatorname{strco}_{M} S \subset \operatorname{strco}_{M} A$ по теореме 5.2 , откуда $s\left(p, \operatorname{strco}_{M} S\right) \leqslant s\left(p, \operatorname{strco}_{M} A\right)$. С другой стороны,

$$
\begin{aligned}
s\left(p, \operatorname{strco}_{M} S\right) & =s(p, M)-\inf \left\{\sum_{i=1}^{n}\left(s\left(q_{i}, M\right)-s\left(q_{i}, S\right)\right): \sum_{i=1}^{n} q_{i}=p\right\} \\
& \geqslant s(p, M)-\sum_{i=1}^{n}\left(s\left(p_{i}, M\right)-s\left(p_{i}, S\right)\right)=s\left(p, \operatorname{strco}_{M} A\right) .
\end{aligned}
$$

Отсюда следует равенство $s\left(p, \operatorname{strco}_{M} S\right)=s\left(p, \operatorname{strco}_{M} A\right)$, которое вместе с равенством (6.1) доказывает включение $u \in \operatorname{strco}_{M} S$. 
Итак, любая точка границы множества $\operatorname{strco}_{M} A$ содержится в $M$-сильно выпуклой оболочке подмножества из $A$, содержащего не более чем $n$ точек.

Пусть $x \in \operatorname{strco}_{M} A \backslash \partial\left(\operatorname{strco}_{M} A\right)$. Существуют точки $u_{0} \in \partial\left(\operatorname{strco}_{M} A\right)$ и $w_{0} \in A$ такие, что $x \in\left[u_{0}, w_{0}\right]$. Как показано выше, для граничной точки $u_{0}$ найдется множество $S_{0}$, состоящее не более чем из $n$ точек компакта $A$ и такое, что точка $u_{0}$ принадлежит $\operatorname{strco}_{M} S_{0}$. Из теоремы 5.2 получаем

$x \in \operatorname{co}\left\{\left(\operatorname{strco}_{M} S_{0}\right) \cup\left\{w_{0}\right\}\right\} \subset \operatorname{strco}_{M}\left\{\left(\operatorname{strco}_{M} S_{0}\right) \cup\left\{w_{0}\right\}\right\} \subset \operatorname{strco}_{M}\left\{S_{0} \cup\left\{w_{0}\right\}\right\}$.

2. Пусть $\operatorname{int}(M \stackrel{*}{*} A)=\varnothing$. Без ограничения общности будем считать, что $0 \in \operatorname{ri}(\operatorname{co} A) \subset M$ и $0 \in \operatorname{int} M$. Пусть число $\lambda \in(0,1)$. Тогда $\operatorname{co}(\lambda A) \subset \operatorname{co~} A$, откуда следует, что $M \stackrel{*}{A} \subset M \stackrel{*}{\lambda} A$. С другой стороны, поскольку разность Минковского-Понтрягина является полунепрерывным сверху многозначным отображением (см., например, [19; гл. $1, \S 4$, теорема 3$])$, то получаем, что $h(M \stackrel{*}{ } \lambda A, M \stackrel{*}{*} A) \rightarrow 0$ при $\lambda \rightarrow 1-0$. Отсюда, воспользовавшись формулой для опорной функции $M$-сильно вьпуклой оболочки (см. теорему 5.1), получим, что при $\lambda \rightarrow 1$

$$
h\left(\operatorname{strco}_{M}(\lambda A), \operatorname{strco}_{M} A\right) \rightarrow 0 .
$$

Кроме того, очевидно, что

$$
\operatorname{int}(M * \lambda A) \neq \varnothing, \quad \lambda \in(0,1) .
$$

Фиксируем $x \in \operatorname{strco}_{M} A, x \notin A$, и последовательность $\lambda_{k} \in(0,1)$, сходящуюся к числу 1 . В силу (6.2) сушествует последовательность $x_{k} \in \operatorname{strco}_{M}\left(\lambda_{k} A\right)$, сходяшаяся к $x$. В силу (6.3) и п. 1 доказательства теоремы для каждого $k=1,2, \ldots$ найдутся $\left\{u_{i k}\right\}_{i=1}^{n+1} \subset \lambda_{k} A$ такие, что $x_{k} \in \operatorname{strco}_{M}\left[\bigcup_{i=1}^{n+1}\left\{u_{i k}\right\}\right]$. Без ограничения обшности найдутся $u_{i} \in A, i=1, \ldots, n+1$, такие, что для каждого $i$ последовательность $u_{i k} \rightarrow u_{i}$ при $k \rightarrow \infty$.

Как показано в [19; гл. $1, \S 7$, теорема 3$]$, операция непустого пересечения фиксированного числа строго выпуклых множеств непрерывна, откуда следует, что $h\left(M * \bigcup_{i=1}^{n+1}\left\{u_{i k}\right\}, M * \bigcup_{i=1}^{n+1}\left\{u_{i}\right\}\right) \rightarrow 0$. Так как, кроме того, по определению сильно выпуклой оболочки для каждого номера $k$ справедливо равенство

$$
\operatorname{strco}_{M}\left[\bigcup_{i=1}^{n+1}\left\{u_{i k}\right\}\right]+\left(M *\left[\bigcup_{i=1}^{n+1}\left\{u_{i k}\right\}\right]\right)=M,
$$

то в итоге получаем, что $h\left(\operatorname{strco}_{M}\left[\bigcup_{i=1}^{n+1}\left\{u_{i k}\right\}\right], \operatorname{strco}_{M}\left[\bigcup_{i=1}^{n+1}\left\{u_{i}\right\}\right]\right) \rightarrow 0$ при $k \rightarrow \infty$. Последнее эквивалентно тому, что для любого $\varepsilon>0$ найдутся достаточно большие $k$, при которых

$$
x \in x_{k}+B_{\varepsilon}(0) \subset \operatorname{strco}_{M}\left[\bigcup_{i=1}^{n+1}\left\{u_{i k}\right\}\right]+B_{\varepsilon}(0) \subset \operatorname{strco}_{M}\left[\bigcup_{i=1}^{n+1}\left\{u_{i}\right\}\right]+B_{2 \varepsilon}(0) .
$$

Отсюда следует, что $x \in \operatorname{strco}_{M}\left[\bigcup_{i=1}^{n+1}\left\{u_{i}\right\}\right]$. 


\section{§ 7. Обобщение теоремы Крейна-Мильмана}

Напомним некоторые нужные нам свойства шаров и проекций в гильбертовом пространстве (см., например, [18], [21], [22]).

ПРЕДЛОЖЕНИЕ 7.1. Пусть $R>r>0, z \in \mathscr{H},\|z\|=R-r$. Тогда $B_{r}(z) \subset$ $B_{R}(0), \partial B_{R}(0) \cap B_{r}(z)=\{z R /\|z\|\} u\left(B_{R}(0)\right)(p)=p R$ для любого $p \in \partial B_{1}(0)$.

Для любого начального вектора $z \in \mathscr{H}$ и произвольного направления $q \in$ $\partial B_{1}(0)$ определим луч по формуле $l(z, q)=\{z+\lambda q: \lambda \geqslant 0\}$.

ПРЕДЛОЖЕНИЕ 7.2. Пусть $В \subset \mathscr{H}$ - выпуклое замкнутое множество, $x \notin B$. Пусть $y-$ проекиия точки $x$ на $B$, определим $p=(x-y) /\|x-y\|$. Тогда р будет нормальным вектором к множеству $B$ в точке у. Обратно, если $p \in \partial B_{1}(0)$ - нормальный вектор к множеству $B$ в точке $y$, то для любой точки $x \in l(y, p)$ проекцией точки $x$ на $B$ будет $y$.

ОПРЕДЕЛЕНИЕ 7.1. Пусть замкнутое множество $A$ из $\mathscr{H}$ таково, что $A \subset B_{\rho}(a)$, где $a \in \mathscr{H}$ и $0<\rho<R$. Тогда точка $x \in A$ называется $R$-сильно выступающей точкой множества $A$, если сушествует точка $z \in \mathscr{H}$ такая, что $A \subset B_{R}(z)$ и $\partial B_{R}(z) \cap A=\{x\}$. Множество всех $R$-сильно выступающих точек множества $A$ будем обозначать через $\exp _{R} A$.

Введем еше одно полезное обозначение:

$$
\partial B_{R}(z, q, \lambda)=\{x:\|x-(z-\lambda q)\|=R,\langle x-(z-\lambda q), q\rangle \geqslant 0\} .
$$

Лемма 7.1. Пусть $A \subset \mathscr{H}-$ компакт, $A \subset B_{r}(z)$, әде $0<r<R$. Тогда множество $\exp _{R} A \neq \varnothing$, причем

$$
\forall q \in \partial B_{1}(0) \exists \lambda \geqslant 0: \exp _{R} A \cap \partial B_{r}(z, q, \lambda) \neq \varnothing \quad u \quad A \subset B_{r}(z-\lambda q) .
$$

ДокАЗАТЕЛЬСТво. Фиксируем $q \in \partial B_{1}(0)$. Пусть

$$
\lambda=\sup \left\{\mu: A \subset B_{r}(z-\mu q)\right\} .
$$

Легко видеть, что $\lambda \in[0,+\infty)$.

Пусть $\lambda_{i}$ - максимизируюшая последовательность для супремума в определении $\lambda$, тогда

$$
\left\|x-z+\lambda_{i} q\right\| \leqslant r \quad \forall x \in A,
$$

откуда в пределе

$$
\|x-z+\lambda q\| \leqslant r \quad \forall x \in A .
$$

Итак, супремум в определении $\lambda$ достигается.

Считаем без ограничения обшности, что $z-\lambda q=0$.

Допустим, что $\partial B_{r}(z, q, \lambda) \cap A=\varnothing$. По теореме о топологической отделимости компакта и замкнутого множества (см. [18; теорема 1.10$])$ получаем, что найдется $\varepsilon>0$ такое, что

$$
\left(A+B_{\varepsilon}(0)\right) \cap\left(\partial B_{r}(z, q, \lambda)+B_{\varepsilon}(0)\right)=\varnothing .
$$


Покажем, что

$$
A \subset B_{r}(-\varepsilon q)
$$

Пусть $x \in A \subset B_{r}(0)$ и $\mu=\mu(x) \geqslant 0$ :

$$
x+\mu q \in \partial B_{r}(0)
$$

Тогда $\langle x+\mu q, q\rangle \geqslant 0$. Если это не так, то $\langle x, q\rangle<-\mu \leqslant 0$. Отсюда

$$
\mu^{2}+2 \mu\langle x, q\rangle=r^{2}-\|x\|^{2} \geqslant 0
$$

что влечет $-\mu^{2}>0$. Последнее неравенство явно неверно, откуда $\langle x+\mu q, q\rangle \geqslant 0$. Поэтому $x+\mu q \in \partial B_{r}(0, q, 0)$. Так как из формулы (7.1) следует, что $\mu \geqslant \varepsilon$ для любого $x \in A$, то $A+\varepsilon q \subset B_{r}(0)$, откуда следует (7.2). Но формула (7.2) противоречит максимальности $\lambda$, следовательно, допушение $\partial B_{r}(z, q, \lambda) \cap A=\varnothing$ неверно.

Итак, найдется $x \in \partial B_{r}(z, q, \lambda) \cap A$. Пусть $p$ - единичный нормальный вектор к множеству $B_{r}(0)$ в точке $x \in \partial B_{r}(0)$; легко видеть, что $p=x /\|x\|$. По предложению 7.1 получаем:

$$
\{x\} \in \partial B_{R}(x-p R) \cap A \subset \partial B_{R}(x-p R) \cap B_{r}(0)=\{x\} .
$$

Кроме того,

$$
A \subset B_{r}(0) \subset B_{R}(x-p R)
$$

Tеорема 7.1. Пусть $A \subset \mathscr{H}$ - компакт, $A \subset B_{\rho}\left(z_{1}\right), \rho<R . \quad$ Тогда $A \subset \operatorname{strco}_{R}\left(\exp _{R} A\right)$.

Доказательство. Пусть $B=\operatorname{strco}_{R}\left(\exp _{R} A\right)$. Через $P_{M}$ обозначим проектор на выпуклое замкнутое множество $M$.

Допустим, что сушествует $x \in A \backslash B$. Пусть $y=P_{B} x \neq x, \varepsilon=\|x-y\|>0$; по предложению $7.2 p=(x-y) / \varepsilon$ - нормальньй вектор к множеству $B$ в точке $y$. В силу опорного принципа для шаров $B \subset B_{R}(z)$, где $z=y-p R ; A \subset B_{\rho}\left(z_{1}\right) \subset$ $B_{r}\left(z_{1}\right)$, где число $r>0$ определим из условий

$$
r=\max \left\{\rho, \frac{R+\varepsilon+\sqrt{(R-\varepsilon)^{2}-\varepsilon^{2}}}{2}\right\} \text { при } R \geqslant 2 \varepsilon ; \quad r=\rho \text { при } R<2 \varepsilon .
$$

Отметим, что $r<R, z \neq z_{1}$. Так как $A \subset \operatorname{strco}_{R} A \subset B_{r}\left(z_{1}\right)$, то $[x, y] \subset B_{r}\left(z_{1}\right)$.

1. Пусть $q=\left(z_{1}-z\right) /\left\|z_{1}-z\right\|$. Отметим, что $\left\|x-z_{1}\right\| \leqslant r$. Введем $a=z_{1}+$ $\left\|z_{1}-x\right\| q \in B_{r}\left(z_{1}\right)$,

$$
\|a-z\|=\left\|z-z_{1}\right\|+\left\|x-z_{1}\right\| \geqslant\|x-z\|>R
$$

откуда $a \notin B_{R}(z)$. Кроме того, $P_{B_{R}(z)} a=z+q R$. Так как $\|x-y\|=\|x-z\|-R$, a $\|a-(z+q R)\|=\|a-z\|-R$, то

$$
\|a-(z+q R)\| \geqslant\|x-y\|=\varepsilon .
$$


Поскольку $\langle q, z+q R\rangle=s\left(q, B_{R}(z)\right)$, то $P_{B_{R}(z)} l(z+q R, q)=\{z+q R\}$ по лемме 7.2. Так как $B_{R}(z) \cap B_{r}\left(z_{1}\right) \neq \varnothing$ и $\left\|z-z_{1}\right\| \geqslant R-r$, то $\left\|z-z_{1}\right\| \in[R-r, R+r]$, откуда $\left\|z-z_{1}+q R\right\| \leqslant r$, т.e. $z+q R \in B_{r}\left(z_{1}\right)$.

В итоге, для любого шара $B_{r}\left(z_{2}\right)$, для которого справедливо включение $A \subset$ $B_{r}\left(z_{2}\right)$ и $z_{2} \in l(z, q)$, отрезок $[z+q R, z+q(R+\varepsilon)]$ лежит в $B_{r}\left(z_{2}\right)$.

2. По лемме 7.1 найдется $\lambda \geqslant 0$ такое, что $\partial B_{r}\left(z_{1}, q, \lambda\right) \cap \exp _{R} A \neq \varnothing$ и $A \subset$ $B_{r}(z-\lambda q)$. Пусть без ограничения обшности $z-\lambda q=0$. В силу п. 1 доказательства $[z+q R, z+q(R+\delta)] \subset B_{r}(0), z+q(R+\delta) \in \partial B_{r}(0)$ и $\delta \geqslant \varepsilon$. Пусть $u \in \partial B_{r}(0, q, 0)$. Это значит, что $\|u\|=r$ и $\langle u, q\rangle \geqslant 0$. Так как $z+(R+\delta) q=r q$, то $z=-(R-r+\delta) q$. Следовательно,

$$
\|u-z\|^{2}-R^{2} \geqslant 2 r^{2}-2 r(R+\varepsilon)+2 \varepsilon R+\varepsilon^{2}>0 .
$$

Таким образом, $\partial B_{r}(0, q, 0) \cap B_{R}(z)=\varnothing$. Итак, сушествует $u$ такое, что

$$
u \in\left(\partial B_{r}(0, q, 0) \cap \exp _{R} A\right) \backslash B_{R}(z) \text { и } \exp _{R} A \subset B_{R}(z) .
$$

Полученное противоречие показьвает, что $A \subset \operatorname{strco}_{R}\left(\exp _{R} A\right)$.

ОПреДЕЛЕНИЕ 7.2. Пусть замкнутое множество $A \subset \mathscr{H}$ и числа $r>0, R>0$ таковы, что $B_{r}(0)$ * $A \neq \varnothing$ и $R \geqslant r$. Непустое подмножество $V \subset A$ назовем $B_{R}(0)$-сильно крайним (или просто $R$-сильно крайним) множеством множества $A$, если ни одна точка $x$ из $V$ не содержится в $R$-сильно выпуклой оболочке любой пары точек $y_{1}, y_{2}$ из $A$, которые отличны от $x$ и удовлетворяют условию $\left\{y_{1}, y_{2}\right\} \not \subset V$.

Точку $x$ назовем $R$-сильно крайней точкой множества $A$, если одноточечное множество $\{x\}$ является $R$-сильно крайним подмножеством множества $A$.

Множество всех $R$-сильно крайних точек множества $A$ будем обозначать через $\operatorname{extr}_{R} A$.

ЛЕмма 7.2. Для любого множества $А \subset \mathscr{H}$ справедливо включение $\exp _{R} A \subset \operatorname{extr}_{R} A$.

ДокаЗАТЕЛЬСтво. Если $\exp _{R} A=\varnothing$, то утверждение очевидно.

Пусть $x \in \exp _{R} A$. Тогда существует шар $B_{R}\left(z_{0}\right)$ такой, что

$$
A \subset B_{R}\left(z_{0}\right) \quad \text { и } \partial B_{R}\left(z_{0}\right) \cap A=\{x\} .
$$

Фиксируем произвольные $y$ и $z$ из $A$, отличные от $x$. Из формулы (7.3) следует, что $\{y, z\} \subset \operatorname{int} B_{R}\left(z_{0}\right)$. Пусть $p \in \partial B_{1}(0)$ - нормальньй вектор к $B_{R}\left(z_{0}\right)$ в точке $x$. Тогда найдется $\varepsilon>0$ такое, что

$$
\{y, z\} \subset B_{R}\left(z_{0}-\varepsilon p\right), \quad x \notin B_{R}\left(z_{0}-\varepsilon p\right) .
$$

Так как по определению сильно выпуклой оболочки $\operatorname{strco}_{R}\{y, z\} \subset B_{R}\left(z_{0}-\varepsilon p\right)$, то

$$
x \notin \operatorname{strco}_{R}\{y, z\} .
$$

Teоpema 7.2. Пусть компакт $A \subset \mathscr{H}$ таков, ито $A \subset B_{\rho}\left(z_{1}\right)$, и пусть $R>\rho$. Тогда справедливо включение $A \subset \operatorname{strco}_{R}\left(\operatorname{extr}_{R} A\right)$.

Доказательство очевидно следует из леммы 7.2 и теоремы 7.1. 


\section{§8. О новых липшицевых селекторах}

Одним из наиболее известных классов непрерывных по Липшицу однозначных селекторов многозначных отображений с выпуклыми и компактными значениями из $\mathbb{R}^{n}$ является класс селекторов, получаемых выбором центра Штейнера [23] в каждом значении многозначного отображения. В этом параграфе показано, как можно получить другие непрерьвные по Липшицу селекторы многозначного отображения, используя центр Штейнера и $B_{R}(0)$-сильно вьпуклую оболочку значений данного многозначного отображения.

Скажем, что функция $f$, действуюшая из множества выпуклых компактов из $\mathbb{R}^{n}$ в пространство $\mathbb{R}^{n}$, называется селектором выпуклых компактов, если для любого выпуклого компакта $A$ справедливо включение $f(A) \in A$.

ОПРЕДЕЛЕНИЕ 8.1. Центром Штейнера вьпуклого компакта $A \subset \mathbb{R}^{n}$ (см. [23] и библиографию в $[24],[25])$ называется точка $s(A) \in \mathbb{R}^{n}$, определяемая по формуле

$$
s(A)=\frac{n}{S_{1}} \int_{\partial B_{1}(0)} s(q, A) q d q, \quad \text { где } \quad S_{1}=\int_{\partial B_{1}(0)} d q .
$$

Известно (см. [24; $\S 3])$, что центр Штейнера $s(A)$ выпуклого компакта $A$ принадлежит этому компакту, т.е. является селектором выпуклых компактов из $\mathbb{R}^{n}$, при этом данный селектор удовлетворяет условию Липшица (иначе говорят: является непрерывным по Липшицу), если компакты рассматривать как элементы метрического пространства с метрикой Хаусдорфа $h(\cdot, \cdot)$. Это означает, что для любых выпуклых компактов $A_{1}$ и $A_{2}$ выполнено неравенство

$$
\left\|s\left(A_{1}\right)-s\left(A_{2}\right)\right\| \leqslant L(n) h\left(A_{1}, A_{2}\right), \quad \text { где } L(n)=\frac{2 \Gamma\left(\frac{n}{2}+1\right)}{\sqrt{\pi} \Gamma\left(\frac{n+1}{2}\right)} .
$$

Известно, что константа Липшица в (8.2), имеющая порядок $\sqrt{n}$, в общем случае является неулучшаемой.

ОПРЕДЕЛЕНИЕ 8.2. Скажем, что $\Omega_{r}$ есть семейство выпуклых компактов из $\mathbb{R}^{n}$, равномерно ограниченных константой $r>0$, если для любого компакта $A \in \Omega_{r}$ найдется точка $x \in \mathbb{R}^{n}$ такая, что $A \subset B_{r}(x)$.

ОПРЕДЕЛЕНИЕ 8.3. Пусть числа $r>0, R>0$ таковы, что $r<R$. Для всякого компакта $A \in \Omega_{r}$ определим точку $p_{R}(A) \in \mathbb{R}^{n}$ следуюшим образом:

$$
p_{R}(A)=-\frac{n}{S_{1}} \int_{\partial B_{1}(0)} \operatorname{co}(R\|q\|-s(q, A)) q d q .
$$

Теорема 8.1. Пусть фиксированы числа $r, R, 0<r<R$, и пусть $\Omega_{r}$ есть семейство выпуклых компактов из $\mathbb{R}^{n}$, равномерно ограниченных константой $r>0$. Тогда справедливо включение

$$
p_{R}(A) \in A \quad \forall A \in \Omega_{r}
$$


и функция $A \rightarrow p_{R}(A)$ является непрерьвной по Липшицу функцией на $\Omega_{r}$, т.е. для любых компактов $A_{1}, A_{2} \in \Omega_{r}$ выполнено неравенство

$$
\left\|p_{R}\left(A_{1}\right)-p_{R}\left(A_{2}\right)\right\| \leqslant L(n, R, r) h\left(A_{1}, A_{2}\right),
$$

əде $L(n, R, r)=L(n) L(R, r)$, причем $L(n)$ определена в (8.2), а $L(R, r)$ - в (5.8).

Для доказательства этой основной теоремы нам потребуются некоторые специальные обозначения и результаты.

Фиксируем числа $r, R, 0<r<R$, и некоторое $R$-сильно выпуклое множество $A \subset \mathbb{R}^{n}$ такое, что $B_{r}(0) \stackrel{*}{*} A \neq \varnothing$.

Фиксируем произвольный вектор $p$ из множества $\partial B_{1}(0)$.

Дугу окружности радиуса $R$ с центром в точке $a \in \mathbb{R}^{n}$ длины меньше $\pi R$ и концами в точках $y_{1}, y_{2}$ будем обозначать через $D_{R}(a)\left[y_{1}, y_{2}\right]$.

Обозначим

$$
\begin{gathered}
H_{p}^{\circ}=\left\{x \in \mathbb{R}^{n}:\langle p, x\rangle=s\left(p, \overline{\operatorname{co}}\left(\operatorname{extr}_{R} A\right)\right)\right\}, \\
H_{p}^{-}=\left\{x \in \mathbb{R}^{n}:\langle p, x\rangle \leqslant s\left(p, \operatorname{co}\left(\operatorname{extr}_{R} A\right)\right)\right\}, \quad H_{p}^{+}=\overline{\mathbb{R}^{n} \backslash H_{p}^{-}}, \\
A_{p}^{\circ}=A \cap H_{p}^{\circ}, \quad A_{p}^{+}=A \cap H_{p}^{+}, \quad A_{p}^{-}=A \cap H_{p}^{-} .
\end{gathered}
$$

Ясно, что $A_{p}^{\circ}$ непусто. Без ограничения общности будем считать, что $0 \in A_{p}^{\circ}$.

Введем отображение $g_{p}: \mathbb{R}^{n} \rightarrow \mathbb{R}^{n}$, обеспечивающее отражение пространства относительно гиперплоскости $H_{p}^{\circ}$, т.е.

$$
g_{p}(x)=x-2 p\langle p, x\rangle \quad \forall x \in \mathbb{R}^{n} .
$$

ЛЕмма 8.1. В обозначениях (8.6)-(8.8) справедливо равенство

$$
H_{p}^{+} \cap \operatorname{strco}_{R} A_{p}^{\circ}=A_{p}^{+} .
$$

ДокаЗАТЕЛЬСтво. Заметим, что из включения $A_{p}^{\circ} \subset A$ следует включение $H_{p}^{+} \cap \operatorname{strco}_{R} A_{p}^{\circ} \subset H_{p}^{+} \cap A=A_{p}^{+}$. Докажем обратное включение.

Пусть вначале $z \in \partial A_{p}^{+} \backslash H_{p}^{\circ}$. Найдется нормальный вектор $\bar{p} \in \partial B_{1}(0)$ такой, что вьполняется равенство $\langle\bar{p}, z\rangle=s(\bar{p}, A)$.

Определим точку $a=z-\bar{p} R$ и множество $C=A \cap \partial B_{R}(a)$. Отметим, что в силу леммы 2 из [9] это множество $C$ является $R$-сильно крайним подмножеством множества $A$. Это значит, что любая дуга окружности радиуса $R$ длины не более $\pi R$, проходящая через $z \in C$, с концами в множестве $A$, отличными от $z$, лежит на поверхности сферы $\partial B_{R}(a)$. С другой стороны, так как $z \in A_{p}^{+}, \operatorname{aextr}_{R} A \subset A_{p}^{-}$, т.е. $z$ не является $R$-сильно крайней точкой множества $A$, то такая дуга сушествует. Пусть дуга $D_{R}(a)\left[y_{1}, y_{2}\right] \subset C$ такова, что $z \in D_{R}(a)\left[y_{1}, y_{2}\right]$ и $y_{1} \neq z, y_{2} \neq z$, причем эта дуга максимальной длины, т.е. она не может быть продолжена в $C$.

Рассмотрим конус

$$
K_{C}=\{a+\lambda(x-a): x \in C, \lambda \geqslant 0\} .
$$


Нетрудно показать, что $K_{C}$ является выпуклым конусом. Так как для точки $z$ дуга $D_{R}(a)\left[y_{1}, y_{2}\right]$ выбрана максимально допустимой длины, то $y_{1}, y_{2} \in \partial K_{C}$. Для каждой из концевых точек проведем аналогичные исследования. Сделаем это на примере точки $y_{1}$.

Если точка $y_{1}$ является $R$-сильно крайней точкой множества $A$, то останавливаемся. Допустим, что это не так. По теореме об отделимости существует вектор $p_{1} \in \partial B_{1}(0)$, отделяющий $y_{1}$ от $K_{C}$, т.е.

$$
\left\langle p_{1}, y_{1}\right\rangle=\left\langle p_{1}, a\right\rangle \geqslant\left\langle p_{1}, x\right\rangle \quad \forall x \in K_{C}
$$

Пусть $\Pi_{1}=\left\{x \in \mathbb{R}^{n}:\left\langle p_{1}, x-a\right\rangle=0\right\}$. Рассмотрим множество $C_{1}=C \cap \Pi_{1}$ и покажем, что $C_{1}$ также является $R$-сильно крайним подмножеством $A$.

Допустим противное, т.е. пусть существуют точки $x \in C_{1}$ и $e_{1}, e_{2} \in C$ такие, что $e_{1}$ не принадлежит $C_{1}$, а $x \in D_{R}(a)\left[e_{1}, e_{2}\right]$ и $x \neq e_{2}$. Тогда существуют числа $\lambda_{1}>0$ и $\lambda_{2}>0$ такие, что $x=a+\lambda_{1}\left(e_{1}-a\right)+\lambda_{2}\left(e_{2}-a\right)$. Так как $\left\langle p_{1}, e_{1}-a\right\rangle<0$, $\mathrm{a}\left\langle p_{1}, e_{2}-a\right\rangle \leqslant 0$, то в итоге имеем

$$
\left\langle p_{1}, x\right\rangle=\left\langle p_{1}, a\right\rangle+\lambda_{1}\left\langle p_{1}, e_{1}-a\right\rangle+\lambda_{2}\left\langle p_{1}, e_{2}-a\right\rangle<\left\langle p_{1}, a\right\rangle
$$

Отсюда следует, что $x \notin C_{1}$, т.е. получаем противоречие. Итак, $C_{1}$ также является $R$-сильно крайним подмножеством $A$, причем его размерность как минимум на единицу менњше размерности $C$.

В итоге, так как $y_{1}$ не является $R$-сильно крайней точкой множества $A$, существует другая дуга $D_{R}(a)\left[y_{1}^{1}, y_{2}^{1}\right]$ в $C_{1}$ максимальной длины такая, что $y_{1} \in D_{R}(a)\left[y_{1}^{1}, y_{2}^{1}\right]$ и $y_{i}^{1} \neq y_{1}, i=1,2$.

Опять исследуем оба конца дуги на примере одного из них, а именно $y_{1}^{1}$. Для этой точки $y_{1}^{1}$ получили задачу построения дуги максимальной длины, аналогичную задаче для $y_{1}$, но в аффиннном многообразии $\Pi_{1}$, которое имеет размерность $n-1$.

Таким образом мы получаем каскад дуг, лежащих на сфере $\partial B_{R}(a)$, заканчивающийся дугами, концы которых являются $R$-сильно крайними точками множества $A$.

Поскольку $\operatorname{extr}_{R} A \subset A_{p}^{-}$, то точки, соответствующие концам дуг, лежат в $A_{p}^{-}$. Пусть множество $\left\{x_{i}\right\}_{i=1}^{m}-$ это точки, в которых дуги каскада пересекают множество $A_{p}^{\circ}$. Как показано в [7], все дуги окружностей радиуса $R$ длины не более $\pi R$ с концами в точках $\left\{x_{i}\right\}_{i=1}^{m}$ целиком лежат в $\operatorname{strco}_{R} A_{p}^{\circ}$, кроме того, любая дуга окружности радиуса $R$ длины не более $\pi R$ с концами в $\operatorname{strco}_{R} A_{p}^{\circ}$ целиком лежит в $\operatorname{strco}_{R} A_{p}^{\circ}$. Но отсюда следует, что $z \in \operatorname{strco}_{R} A_{p}^{\circ}$.

Учитьвая очевидное равенство $\left(\operatorname{strco}_{R} A_{p}^{\circ}\right) \cap H_{p}^{\circ}=A_{p}^{+} \cap H_{p}^{\circ}$, получаем, что граничные точки множеств $H_{p}^{+} \cap \operatorname{strco}_{R} A_{p}^{\circ}$ и $A_{p}^{+}$совпадают, а так как эти множества вьпуклы, то сами они тоже совпадают. Итак, (8.10) доказано.

ЛЕмма 8.2. Для отображсения (8.9) справедливо включение

$$
g_{p}\left(A_{p}^{+}\right) \subset A_{p}^{-}
$$


ДокАЗАТЕЛЬСТво. Из включения $A_{p}^{\circ} \subset B_{R}(x)$ и определения $g_{p}$ по (8.9) очевидно следует включение $A_{p}^{\circ} \subset B_{R}\left(g_{p}(x)\right)$ для любого $x \in \mathbb{R}^{n}$. Поэтому $g_{p}\left(\operatorname{strco}_{R} A_{p}^{\circ}\right)=\operatorname{strco}_{R} A_{p}^{\circ}$. Кроме того, $g_{p}\left(H_{p}^{+}\right)=H_{p}^{-}$. В силу включения $\operatorname{strco}_{R} A_{p}^{\circ} \subset A$ и по лемме 8.1 получаем

$$
g_{p}\left(A_{p}^{+}\right)=\left(\operatorname{strco}_{R} A_{p}^{\circ}\right) \cap H_{p}^{-} \subset A \cap H_{p}^{-}=A_{p}^{-}
$$

ТЕОРема 8.2. Пусть числа $r, R, 0<r<R, u R$-сильно выпуклое множсество $A \subset \mathbb{R}^{n}$ таковьи, что $B_{r}(0) \stackrel{*}{*} \neq \varnothing$. Тогда иентр ШІтейнера $s(A)$ удовлетворяет включению

$$
s(A) \in \overline{\operatorname{co}}\left(\operatorname{extr}_{R} A\right)
$$

ДоказАТЕЛЬСтво. Прежде всего для произвольного фиксированного $p \in$ $\partial B_{1}(0)$ в обозначениях (8.6)-(8.9) докажем включение

$$
s(A) \in H_{p}^{-} .
$$

Обозначим

$$
B_{1, p}^{+}(0)=\left\{q \in B_{1}(0): 0 \leqslant\langle p, q\rangle\right\}, \quad B_{1, p}^{-}(0)=\left\{q \in B_{1}(0):\langle p, q\rangle \leqslant 0\right\} .
$$

Покажем, что

$$
s(q, A) \leqslant s\left(g_{p}(q), A\right) \quad \forall q \in \partial B_{1, p}^{+}(0) .
$$

Фиксируем $q \in B_{1, p}^{+}(0)$, причем $\|q\|=1$. Рассмотрим точку $x_{q} \in A(q)$, т.е. такую точку из $A$, для которой справедливо равенство $\left\langle q, x_{q}\right\rangle=s(q, A)$. Разложим точки $q$ и $x_{q}$ в прямые суммы точек вида

$$
q=q^{\perp}+\lambda_{q} p, \quad x_{q}=x_{q}^{\perp}+\mu_{x q} p
$$

где $\left\langle p, q^{\perp}\right\rangle=0$ и $\left\langle p, x_{q}^{\perp}\right\rangle=0$, a $\lambda_{q}, \mu_{x q} \in \mathbb{R}$.

Так как $q \in B_{1, p}^{+}(0)$, а $\langle p, q\rangle=\lambda_{q}$, то $\lambda_{q} \geqslant 0$. Заметим, что в силу определения симметрии (8.9) получаем $g_{p}(q)=q^{\perp}-\lambda_{q} p$ и $g_{p}\left(x_{q}\right)=x_{q}^{\perp}-\mu_{x q} p$. Для $x_{q}$ возможна одна из двух ситуаций: либо $x_{q} \in \partial A_{p}^{+}$, либо $x_{q} \in \partial A_{p}^{-}$.

Допустим, что $x_{q} \in \partial A_{p}^{+}$. Тогда по лемме $8.2 g_{p}\left(x_{q}\right) \in A_{p}^{-}$и

$$
\begin{aligned}
s(q, A) & =\left\langle q, x_{q}\right\rangle=\left\langle q^{\perp}+\lambda_{q} p, x^{\perp}+\mu_{x q} p\right\rangle \\
& =\left\langle q^{\perp}, x^{\perp}\right\rangle+\lambda_{q} \mu_{x q}=\left\langle q^{\perp}-\lambda_{q} p, x^{\perp}-\mu_{x q} p\right\rangle \leqslant s\left(g_{p}(q), A\right) .
\end{aligned}
$$

Допустим, что $x_{q} \in \partial A_{p}^{-}$. Это означает, что $\mu_{x q} \leqslant 0$. Поэтому

$$
\begin{aligned}
s(q, A) & =\left\langle q, x_{q}\right\rangle=\left\langle q^{\perp}+\lambda_{q} p, x^{\perp}+\mu_{x q} p\right\rangle \\
& =\left\langle q^{\perp}, x^{\perp}\right\rangle+\lambda_{q} \mu_{x q} \leqslant\left\langle q^{\perp}-\lambda_{q} p, x^{\perp}+\mu_{x q} p\right\rangle \leqslant s\left(g_{p}(q), A\right) .
\end{aligned}
$$


Итак, неравенство (8.15) доказано. Из него получаем

$$
\begin{aligned}
\langle p, s(A)\rangle & =\frac{n}{S_{1}} \int_{\partial B_{1}(0)} s(q, A)\langle q, p\rangle d q \\
& =\frac{n}{S_{1}} \int_{\partial B_{1, p}^{+}(0)} s(q, A)\langle q, p\rangle d q-\frac{n}{S_{1}} \int_{\partial B_{1, p}^{+}(0)} s\left(g_{p}(q), A\right)\langle q, p\rangle d q \leqslant 0,
\end{aligned}
$$

т.е. справедливо включение (8.14). Так как (8.14) доказано для произвольного вектора $p$ из $\partial B_{1}(0)$, то

$$
s(A) \in \bigcap_{|p|=1} H_{p}^{-}=\overline{\operatorname{co}}\left(\operatorname{extr}_{R} A\right)
$$

что и требовалось доказать.

Лемма 8.3. Пусть множсество $A \subset \mathbb{R}^{n}$ является $R$-сильно выпукльм, причем $B_{r}(0) \stackrel{*}{A} \neq \varnothing$, где $r<R$. Виберем точку $z \in \operatorname{extr}_{R} A$ и компакт $B \subset A \backslash\{z\}$. Тогда $z \notin \operatorname{strco}_{R} B$.

ДоказАтельство. Допустим, что $z \in \operatorname{strco}_{R} B$. Существует нормальный вектор $p \in \partial B_{1}(0)$ такой, что $\langle p, z\rangle=s(p, A)$. Обозначим $a=z-p R$ и $C=\partial B_{R}(a) \cap A$ и для простоты далее в доказательстве будем полагать, что $a=0$. Определим конус $K_{C}=\{\lambda x: x \in C, \lambda \geqslant 0\}$. Легко показать, что множество $K_{C}$ является вьпуклым острым конусом.

Убедимся в том, что луч $L=\{\lambda z: \lambda \geqslant 0\}$ является крайним лучом (см. [26], [17]) для конуса $K_{C}$.

Допустим противное, тогда сушествуют точки $e_{1}, e_{2} \in C \backslash\{z\}$ и числа $\alpha_{i}>0$, $i=1,2$, такие, что выполнено равенство $z=\alpha_{1} e_{1}+\alpha_{2} e_{2}$, т.е. $z \in D_{R}(0)\left[e_{1}, e_{2}\right]$. Но это противоречит тому, что $z$ является $R$-сильно крайней точкой множества $A$. Итак, луч $L$ является крайним лучом для $K_{C}$.

Так как по допушению $z \in \operatorname{strco}_{R} B$, то по теореме 6.1 найдется набор точек $\left\{x_{i}\right\}_{i=1}^{m}, m \leqslant n+1$, из множества $B$ таких, что $z$ содержится в $R$-сильно вьпуклой оболочке этого набора. Из включения $B \subset A$ следует, что $\left\{x_{i}\right\}_{i=1}^{m} \subset A$.

Пусть $\left\{x_{i_{k}}\right\}_{k=1}^{l}$ суть все точки набора $\left\{x_{i}\right\}_{i=1}^{m}$, которые принадлежат $C=$ $\partial B_{R}(0) \cap A$. Определим множество индексов $I=\{1, \ldots, m\} \backslash\left\{i_{k}\right\}_{k=1}^{l}$. Тогда для каждого $j \in I$ существует число $\varepsilon_{j}>0$ такое, что $x_{j}+\varepsilon_{j} B_{1}(0) \subset B_{R}(0)$.

Рассмотрим конус

$$
K=\operatorname{coneco}\left(\left(\bigcup_{k=1}^{l}\left\{x_{i_{k}}\right\}\right) \cup\{z\}\right)
$$

Это многогранный выпуклый конус, причем из включения $\left\{z, x_{i_{1}}, \ldots, x_{i_{l}}\right\} \subset C$ следует, что $K \subset K_{C}$. Отсюда получаем, что конус $K$ также является острым конусом, а луч $L \subset K$ является крайним лучом для $K$.

Так как для многогранного конуса любой крайний луч является выступающим (см., например, [27; гл. 1, теоремы 4.2 и 4.7]), то найдется вектор $q \in \partial B_{1}(0)$ такой, что $K \cap \Pi_{q}=L$, где $\Pi_{q}$ есть гиперплоскость вида $\Pi_{q}=\left\{x \in \mathbb{R}^{n}:\langle q, x\rangle=0\right\}$. 
Определим числа

$$
\alpha=\min _{1 \leqslant k \leqslant l} \varrho\left(x_{i_{k}}, \Pi_{q}\right), \quad \beta=\min _{j \in I} \varepsilon_{j} \quad \text { и } \gamma=\min \{\alpha, \beta\} .
$$

Очевидно, что $\alpha>0, \beta>0, \gamma>0$. Легко проверить, что все точки $\left\{x_{i}\right\}_{i=1}^{m}$ принадлежат шару $B_{R}(-\gamma q)$. С другой стороны, $\|z+\gamma q\|^{2}=\|z\|^{2}+\gamma^{2}>R^{2}$, т.е. $z$ не принадлежит $B_{R}(-\gamma q)$. По определению сильно выпуклой оболочки это значит, что $z \notin \operatorname{strco}_{R}\left\{\bigcup_{i=1}^{m} x_{i}\right\}$. Полученное противоречие показьвает, что допушение о включении $z \in \operatorname{strco}_{R} B$ неверно.

Теорема 8.3. Пусть числа $r, R, 0<r<R$, и компакт $A \subset \mathbb{R}^{n}$ таковьи, что $B_{r}(0) \stackrel{*}{*} A \neq \varnothing$. Тогда справедливо включение

$$
\operatorname{extr}_{R} A \supset \operatorname{extr}_{R}\left(\operatorname{strco}_{R} A\right)
$$

ДокАЗАТЕЛЬСтво. Так как из включения $A \subset B_{r}(a)$ (при некотором $a$ ) следует, что $\operatorname{strco}_{R} A \subset B_{r}(a)$, и так как $r<R$, то легко убедиться, что множества $\operatorname{extr}_{R} A$ и $\operatorname{extr}_{R}\left(\operatorname{strco}_{R} A\right)$ непусты.

Заметим, что если некоторая точка $z \in \operatorname{extr}_{R}\left(\operatorname{strco}_{R} A\right)$ и $z \in A$, то в силу определения 7.2 эта точка $z$ является $R$-сильно крайней точкой множества $A$. Поэтому для доказательства теоремы достаточно показать, что из включения $z \in \operatorname{extr}_{R}\left(\operatorname{strco}_{R} A\right)$ всегда следует включение $z \in A$.

Пусть $z \in \operatorname{extr}_{R}\left(\operatorname{strco}_{R} A\right)$. По теореме 6.1 сушествует конечное множество точек $\left\{x_{i}\right\}, i=1, \ldots, m$, из $A$, где $m \leqslant n+1$, таких, что $z \in \operatorname{strco}_{R}\left(\bigcup_{i=1}^{m}\left\{x_{i}\right\}\right)$.

Допустим, что $z \notin A$. Тогда $z \neq x_{i}$ при всех $i=1, \ldots, m$. Так как компакт $\bigcup_{i=1}^{m}\left\{x_{i}\right\}$ содержится в $\operatorname{strco}_{R} A$ и не содержит $z$, то по лемме 8.3 получаем, что $z \notin \operatorname{strco}_{R}\left(\bigcup_{i=1}^{m}\left\{x_{i}\right\}\right)$, что неверно. Следовательно, $z \in A$.

ТЕОРема 8.4. Пусть числа $r, R, 0<r<R, u$ выпуклый компакт $A \subset \mathbb{R}^{n}$ таковьи, что $B_{r}(0) \stackrel{*}{*} A \neq \varnothing$. Тогда

$$
s\left(\operatorname{strco}_{R} A\right) \in A .
$$

ДокАЗАТЕЛьство. Применяя теорему 8.2, а затем теорему 8.3, получаем, что

$$
s\left(\operatorname{strco}_{R} A\right) \in \overline{\operatorname{co}}\left(\operatorname{extr}_{R}\left(\operatorname{strco}_{R} A\right)\right) \subset \overline{\operatorname{co}}\left(\operatorname{extr}_{R} A\right) \subset A .
$$

\section{Приведем теперь}

ДоКАЗАТЕЛЬСтво ТЕоРемы 8.1. Легко проверить в силу (8.3) и (5.2), (5.3) справедливость равенства $p_{R}(A)=s\left(\operatorname{strco}_{R} A\right)$. Тогда по теореме 8.4 получаем включение (8.4).

Поскольку операция взятия $R$-сильно выпуклой оболочки является непрерьвной по Липшицу функцией множеств из $\Omega_{r}$ в метрике Хаусдорфа (см. [9], [13] и замечание 5.4) с константой Липшица $L(R, r)$ из (5.8), а центр Штейнера является непрерывным по Липшицу (с константой Липшица $L(n)$ из $(8.2)$ ) селектором выпуклых компактных множеств, то $p_{R}(A)$ как суперпозиция указанных выше отображений является непрерывным по Липшицу селектором выпуклых равномерно ограниченных компактов $\Omega_{r}$ в метрике Хаус дорфа. 


\section{§9. Примеры и контрпримеры}

ПримеР 9.1. Рассмотрим в $\mathbb{R}^{3}$ правильную пирамиду с квадратным основанием вида

$$
M=\operatorname{co}\{(0,0,1),(1,1,0),(-1,1,0),(1,-1,0),(-1,-1,0)\} .
$$

Пусть $A=(M+a) \cap(M-a)$, где $a=(1 / 2,0,0)$. Легко проверить, что

$$
\begin{aligned}
A=\operatorname{co}\{(1 / 2,1,0),(1 / 2,-1,0),(-1 / 2,1,0), \\
(-1 / 2,-1,0),(0,-1 / 2,1 / 2),(0,1 / 2,1 / 2)\} .
\end{aligned}
$$

Пусть $p=(0,0,1)$. Тогда опорное множество $A(p)$ есть отрезок вида

$$
[(0,-1 / 2,1 / 2),(0,1 / 2,1 / 2)]
$$

а опорное множество $M(p)$ состоит из одной точки, т.е. $M(p)=x_{p}^{M}=(0,0,1)$. Выберем точку $x_{p}^{A}=(0,0,1 / 2) \in A(p)$. Легко убедиться в том, что отрезок $A(p)-x_{p}^{A}$ не содержится в множестве $M-x_{p}^{M}$, т.е. не выполнен опорный принцип (следствие 1.1) для данного множества $A$, следовательно, множество $M$ не является порождающим.

ПримеР 9.2. Через $D_{r}(a)[b, c]$ будем обозначать дугу окружности радиуса $r$ длины не более $\pi r$ с концами в точках $b$ и $c$ и центром кривизны в точке $a$. Пусть $M$ есть множество, образованное врашением вокруг оси абсцисс дуги

$$
D_{5}((3,-4,0))[(0,0,0),(6,0,0)] .
$$

Пусть $A=(M+a) \cap(M-a)$, где $a=(0,1 / 2,0)$. Легко видеть, что $A=C_{1} \cup C_{2}$, где

$$
C_{1}=M \cap\{(x, y, z): y \geqslant 1 / 2\}-(0,1 / 2,0),
$$

а $C_{2}$ симметрично $C_{1}$ относительно плоскости $x O z$. Плоская кривая в плоскости $x O z$, ограничиваюшая множество, полученное пересечением $A$ и плоскости $x O z$, гладкая, так как ее сдвиг на вектор $(0,1 / 2,0)$ совпадает с гладкой кривой $\partial M \cap\{(x, y, z): y=1 / 2\}$. Пусть $p=(-1,0,0)$. Тогда $x_{p}^{A}=(3-\sqrt{19} / 2,0,0)$. Касательный конус к множеству $A$ в точке $x_{p}^{A}$ содержит полуплоскость $\{(x, y, z): y=0$, $x \geqslant 0\}$, так как $A$ содержит двумерное подмножество $A \cap\{(x, y, z): y=0\}$, ограниченное гладкой кривой. Касательньй конус к множеству $M$ в точке $x_{p}^{M}=(0,0,0)$ состоит из таких векторов $q \in \mathbb{R}^{3}$, для которых угол между $q$ и вектором $(1,0,0)$ не больше, чем угол между прямой $y=0, z=0$ и $D_{5}((3,-4,0))[(0,0,0),(6,0,0)]$ в точке $(0,0,0)$, т.е. $\arcsin (3 / 5)$. Итак, касательный конус к $M$ в точке $x_{p}^{M}$ является острьм. Отсюда следует, что включение $A \subset M+x_{p}^{A}-x_{p}^{M}$ не выполнено и по следствию 1.1 данное множество $M$ не является порождающим.

Пример 9.3. Пусть $M$ есть симплекс полной размерности в $\mathbb{R}^{n}$, т.е. вьпуклая оболочка $n+1$ точки общего положения. Легко убедиться в том, что $M$ является порождающим множеством, так как всякое множество $A$, полученное как пересечение сдвигов симплекса $M$, есть либо симплекс полной размерности c $(n-1)$-мерными гранями, параллельными $(n-1)$-мерньм граням симплекса $M$ (а значит, подобный симплексу $M$ ), либо точка. В обоих случаях легко указать другой симплекс $B$, подобный симплексу $M$, такой, что $A+B=M$. 
ПримеР 9.4. Пусть $\left\{e_{i}\right\}_{i=1}^{4}$ - ортонормированньй базис в $\mathbb{R}^{4}, e_{0}=0 \in \mathbb{R}^{4}$. Пусть $\lambda_{k}=1 / k$. Рассмотрим последовательность симплексов полной размерности из $\mathbb{R}^{4}$ вида

$$
M_{k}=\operatorname{co}\left\{e_{1}+e_{2}+\lambda_{k} e_{4}, e_{1}-e_{2},-e_{1}-e_{2},-e_{1}+e_{2}, e_{3}\right\}
$$

Пусть $M_{0}=\operatorname{co}\left\{e_{1}+e_{2}, e_{1}-e_{2},-e_{1}-e_{2},-e_{1}+e_{2}, e_{3}\right\}$. Легко видеть, что $M_{k} \rightarrow M_{0}$ при $k \rightarrow \infty$, внутренность $M_{0}$ пуста и нет включения $M_{k} \supset M_{0}$ для всех $k ; M_{0}=$ $M \times\{0\}$, где $M$ - трехмерная правильная четырехугольная пирамида, описанная в примере 9.1, следовательно, $M_{0}$ не является порождающим множеством.

ПримеР 9.5. Пусть $\left\{e_{i}\right\}_{i=1}^{4}$ есть некоторый ортонормированный базис в $\mathbb{R}^{4}$ и $e_{0}=0$. Определим в $\mathbb{R}^{4}$ симплекс полной размерности вида $\Sigma=\operatorname{co}\left\{e_{i}\right\}_{i=0}^{4}$. Как показано в примере 9.3 , множество $\Sigma$ является порождающим множеством. Пусть линейньй оператор $T$ задается матрицей

$$
T=\left(\begin{array}{llll}
1 & 0 & 2 & 2 \\
1 & 2 & 2 & 0 \\
1 & 0 & 0 & 0 \\
0 & 0 & 0 & 0
\end{array}\right)
$$

Тогда легко показать, что $T \Sigma=M \times\{0\}+(1,1,0,0)$, где $M$ есть трехмерная правильная четырехугольная пирамида, которая, как показано в примере 9.1, не является порождающим множеством. Поэтому и $T \Sigma$ также не является порождающим множеством.

ПримеР 9.6. Пусть $\left\{e_{i}\right\}_{i=1}^{3}$ есть ортонормированный базис в $\mathbb{R}^{3}$ и $e_{0}=0$. Рассмотрим тетраэдры, являющиеся порождающими множествами в $\mathbb{R}^{3}$ :

$$
\begin{aligned}
& \Sigma_{1}=\operatorname{co}\left\{e_{0},-e_{1}, e_{2}, \frac{1}{2}\left(e_{2}-e_{1}\right)+\frac{1}{\sqrt{2}} e_{3}\right\} \\
& \Sigma_{2}=\operatorname{co}\left\{e_{0},-e_{1}, e_{2}, \frac{1}{2}\left(e_{2}-e_{1}\right)-\frac{1}{\sqrt{2}} e_{3}\right\} .
\end{aligned}
$$

Определим многогранник $M=\Sigma_{1}+\Sigma_{2}$. Точка 0 есть вершина многогранника $M$, из которой исходят четыре ребра $\left[0,-2 e_{1}\right],\left[0,2 e_{2}\right],\left[0, \frac{1}{2}\left(e_{2}-e_{1}\right) \pm \frac{1}{\sqrt{2}} e_{3}\right]$. Таким образом, точка 0 является вершиной правильной четырехугольной пирамиды

$$
S=\operatorname{co}\left\{0,-e_{1}, e_{2}, \frac{1}{2}\left(e_{2}-e_{1}\right)+\frac{1}{\sqrt{2}} e_{3}, \frac{1}{2}\left(e_{2}-e_{1}\right)-\frac{1}{\sqrt{2}} e_{3}\right\}
$$

такой, что $B_{1 / 2}(0) \cap S=B_{1 / 2}(0) \cap M$. Выберем $p=(1 / \sqrt{2},-1 / \sqrt{2}, 0)$. Тогда $M(p)=\left\{x_{p}^{M}\right\}=\{0\}$. Пусть $X=[a, b]$, где

$$
a=\frac{1}{3}\left(-\frac{3 e_{1}}{2}+\frac{e_{2}}{2}+\frac{e_{3}}{\sqrt{2}}\right), \quad b=\frac{1}{3}\left(-\frac{3 e_{1}}{2}+\frac{e_{2}}{2}-\frac{e_{3}}{\sqrt{2}}\right) .
$$


Определим $Y=M \stackrel{*}{*}$, для которого получим

$$
Y(p)=(S \stackrel{*}{*} X)(p)=\operatorname{co}\left\{\left(\frac{e_{1}}{6}+\frac{e_{2}}{6}+\frac{e_{3}}{3 \sqrt{2}}\right),-\left(\frac{e_{1}}{6}+\frac{e_{2}}{6}+\frac{e_{3}}{3 \sqrt{2}}\right)\right\} .
$$

Выберем $x_{p}^{Y}=0 \in Y(p)$. Легко убедиться в том, что $Y(p) \not \subset M(p)+x_{p}^{Y}-x_{p}^{M}$, т.е. не выполнен опорный принцип (следствие 1.1), и поэтому $M$ не является порождающим множеством.

ПримеР 9.7. Покажем, что константа Липшица оператора $B_{R}(0)$-сильно выпуклой оболочки может оказаться строго больше единицы. Рассмотрим случай, когда шар $B_{R}(0)$ взят на плоскости $\mathbb{R}^{2}$. Фиксируем число $d \in(0, R)$. Рассмотрим в $\mathbb{R}^{2}$ два множества $A_{1}$ и $A_{2}$, где

$$
A_{1}=\left\{(x, y): x^{2}+\left(y+\sqrt{R^{2}-d^{2}}\right)^{2} \leqslant R^{2}, y \geqslant 0\right\}
$$

а множество $A_{2}$ симметрично множеству $A_{1}$ относительно прямой

$$
\left\{(x, y): y=\frac{R-\sqrt{R^{2}-d^{2}}}{2}\right\}
$$

Отметим, что $A_{1} \subset B_{d}(0)$, а $A_{2} \subset B_{d}(b)$, где точка $b \in \mathbb{R}^{2}, b=\left(0, R-\sqrt{R^{2}-d^{2}}\right)$. $\mathrm{C}$ помощью элементарной планиметрии можно получить, что расстояние по Хаусдорфу между $A_{1}$ и $A_{2}$ равняется $h_{1}=\sqrt{R^{2}+d^{2}}-R$. Легко убедиться в том, что $B_{R}(0)$-сильно вьпуклые оболочки множеств $A_{1}$ и $A_{2}$ вычисляются по формулам

$$
\operatorname{strco}_{R} A_{1}=A_{1} \cup\left(-A_{1}\right), \quad \operatorname{strco}_{R} A_{2}=A_{2} \cup\left(A_{1}+b\right) .
$$

Отсюда легко получаем, что расстояние между $B_{R}(0)$-сильно вьпуклыми оболочками множеств $A_{1}$ и $A_{2}$ равняется $h_{2}=R-\sqrt{R^{2}-d^{2}}$. Очевидно, что $h_{2}>h_{1}$, т.е. доказано требуемое утверждение.

\section{Список литературы}

1. Levi F. W. On Helly's theorem and the axioms of convexity // J. Indian Math. Soc. (N.S.). 1951. V. 15. P. 65-76.

2. Klee V.L. (ed. ) Convexity. Proceedings of symposia in pure mathematics. Vol. VIII. Held at the University of Washington Scattle, Washington June 13-15, 1961. Providence, RI: Amer. Math. Soc., 1963.

3. Dolecki S., Kurcyusz S. On $\Phi$-convexity in extremal problems // SIAM J. Control Optim. 1978. V. 16. P. 277-300.

4. Солтан В. П. Введение в аксиоматическую теорию выпуклости. Кишинев: Штиинца, 1984.

5. Ben-Tal A., Ben-Israel A. F-convex functions: properties and applications // Generalized concavity in optimization and economics. Proc. NATO Adv. Study Inst., Vancouver/Can., 1980. Academic Press, 1981. P. 301-334.

6. Singer I. Surrogate conjugate functions and surrogate convexity // Appl. Anal. 1983. V. 16. P. 291-327.

7. Frankowska H., Olech C. R-convexity of the integral of the set-valued functions // Contributions to analysis and geometry. Baltimore, MD: Johns Hopkins Univ. Press, 1981. P. 117-129. 
8. Половинкин E. C. О свойствах сильно вьпуклых множеств // Моделирование процессов управления и обработки информации. М.: Изд-во МФТИ, 1994. С. 182-189.

9. Половинкин Е. С. Сильно выпуклый анализ // Матем. сб. 1996. Т. 187. № 2. С. 103-130.

10. Половинкин E. C. О вьпуклых и сильно выпуклых аппроксимациях множеств // Докл. PAH. 1996. T. 350. № 3. C. 308-311.

11. Polovinkin E. S. On strongly convex sets // Phystech J. 1996. V. 2. № 1. P. 43-59.

12. Половинкин E. C. Обобщение теорем Каратеодори и Крейна-Мильмана для сильн выпуклых множеств // Докл. РАН. 1997. Т. 355. № 2. С. 164-166.

13. Балашов M. В., Половинкин Е. С. Сильно выпуклая оболочка и ее свойства // Некоторые проблемы современной математики и их приложения к задачам физики и механики. М.: Изд-во МФТИ, 1995. С. 27-36.

14. Поляк Б. Т. Введение в оптимизацию. М.: Наука, 1983.

15. Обен Ж.-П., Экланд И. Прикладной нелинейньй анализ. М.: Мир, 1984.

16. Иоффе А. Д., Тихомиров В. М. Теория экстремальных задач. М.: Наука, 1974.

17. Рокафеллар Р. Выпуклый анализ. М.: Мир, 1973.

18. Рудин У. Функциональньй анализ. М.: Мир, 1975.

19. Половинкин E. С. Элементы теории многозначных отображений. М.: Изд-во МФТИ, 1982.

20. Экланд И., Темам Р. Выпуклый анализ и вариационные проблемы. М.: Наука, 1979.

21. Данфорд Н., Швари, Джс. Линейные операторы. Общая теория. М.: ИЛ, 1962.

22. Колмогоров А.Н., Фомин С. В. Элементы теории функций и функционального анализа. М.: Наука, 1972.

23. Steiner J. Von dem Krümmungsschwerpunkte ebener Curven // J. Reine Angew. Math.; 21, 33-63, 101-122, (1840) ou Ges. Werke, Bd. 2 Berlin, G. Reiner (1882), 99-159.

24. Saint Pierre J. Point de Steiner et sections lipschitziennes // Sém. Anal. Convexe. 1985. V. 15. № 7 .

25. Грюнбаум Б. Этюды по комбинаторной геометрии и теории вьпуклых тел. М.: Наука, 1971.

26. Лейхтвейс К. Выпуклые множества. М.: Наука, 1985.

27. Пшеничный Б. Н. Выпуклый анализ и экстремальные задачи. М.: Наука, 1980.

Московский физико-технический институт

E-mail: polovinkin@math.mipt.ru

Поступила в редакцию

18.02.1999 